\title{
Homological vanishing for the Steinberg representation
}

\author{
Avner Ash $\quad$ Andrew Putman* Steven V Sam ${ }^{\dagger}$
}

November 20, 2017

\begin{abstract}
For a field $\mathrm{k}$, we prove that the $i$ th homology of the groups $\mathrm{GL}_{n}(\mathrm{k}), \mathrm{SL}_{n}(\mathrm{k}), \mathrm{Sp}_{2 n}(\mathrm{k})$, $\mathrm{SO}_{n, n}(\mathrm{k})$, and $\mathrm{SO}_{n, n+1}(\mathrm{k})$ with coefficients in their Steinberg representations vanish for $n \geq 2 i+2$.
\end{abstract}

\section{Introduction}

Let $\mathbf{G}$ be a connected reductive group over a field k. A basic geometric object associated to $\mathbf{G}(\mathrm{k})$ is its Tits building. By definition, this is the simplicial complex $\mathcal{T}_{\mathbf{G}}(\mathrm{k})$ whose $i$-simplices are increasing sequences

$$
0 \subsetneq P_{0} \subsetneq \cdots \subsetneq P_{i} \subsetneq \mathbf{G}(\mathrm{k})
$$

of parabolic k-subgroups of $\mathbf{G}(\mathrm{k})$. Letting $r$ be the semisimple k-rank of $\mathbf{G}$, the complex $\mathcal{T}_{\mathbf{G}}(\mathrm{k})$ is $(r-1)$-dimensional, and the Solomon-Tits theorem [Br1, Theorem IV .5.2] says that in fact $\mathcal{T}_{\mathbf{G}}(\mathrm{k})$ is homotopy equivalent to a wedge of $(r-1)$-dimensional spheres. Letting $R$ be a commutative ring, the Steinberg representation of $\mathbf{G}(\mathrm{k})$ over $R$, denoted $\mathrm{St}_{\mathbf{G}}(\mathrm{k} ; R)$, is $\widetilde{\mathrm{H}}_{r-1}\left(\mathcal{T}_{\mathbf{G}}(\mathrm{k}) ; R\right)$. This is one of the most important representations of $\mathbf{G}(\mathrm{k})$; for instance, if $\mathbf{G}$ is any of the classical groups in Theorem 1.1 below (e.g. $\mathbf{G}=\mathrm{SL}_{n}$ ) and $\mathrm{k}$ is a finite field of characteristic $p$, then $\operatorname{St}_{\mathbf{G}}(\mathrm{k} ; \mathbb{C})$ is the unique irreducible representation of $\mathbf{G}(\mathrm{k})$ whose dimension is a positive power of $p$ (see [MalZ], which proves this aside from three small cases that must be checked by hand). See $[\mathrm{H}]$ for a survey of many results concerning the Steinberg representation.

The twisted homology groups $\mathrm{H}_{i}\left(\mathbf{G}(\mathrm{k}) ; \mathrm{St}_{\mathbf{G}}(\mathrm{k} ; R)\right)$ play an interesting role in algebraic K-theory; see [Q, Theorem 3]. If $\mathbf{G}(\mathrm{k})$ is a finite group of Lie type, then $\mathrm{St}_{\mathbf{G}}(\mathrm{k} ; \mathrm{k})$ is a projective $\mathbf{G}(\mathrm{k})$-module (see $[\mathrm{H}]$ ), and thus the homology groups $\mathrm{H}_{i}\left(\mathbf{G}(\mathrm{k}) ; \mathrm{St}_{\mathbf{G}}(\mathrm{k} ; \mathrm{k})\right.$ ) all vanish. However, it is definitely not the case that $\operatorname{St}_{\mathbf{G}}(\mathrm{k} ; R)$ is projective for a general commutative ring $R$, and if $\mathrm{k}$ is an infinite field then $\operatorname{St}_{\mathbf{G}}(\mathrm{k} ; \mathrm{k})$ need not be projective. Our main theorem says that nevertheless for the classical groups, the homology groups $\mathrm{H}_{i}\left(\mathbf{G}(\mathrm{k}) ; \mathrm{St}_{\mathbf{G}}(\mathrm{k} ; R)\right)$ always vanish in a stable range.

Theorem 1.1. Let $\mathrm{G}_{n}$ be either $\mathrm{GL}_{n}, \mathrm{SL}_{n}, \mathrm{Sp}_{2 n}, \mathrm{SO}_{n, n}$, or $\mathrm{SO}_{n, n+1}$. Then for all fields $\mathrm{k}$ and all commutative rings $R$, we have $\mathrm{H}_{i}\left(\mathbf{G}_{n}(\mathrm{k}) ; \mathrm{St}_{\mathbf{G}_{n}}(\mathrm{k} ; R)\right)=0$ for $n \geq 2 i+2$. Furthermore, there exists a surjection $\mathrm{H}_{i}\left(\mathbf{G}_{2 i}(\mathrm{k}) ; \mathrm{St}_{\mathbf{G}_{2 i}}(\mathrm{k} ; R)\right) \rightarrow \mathrm{H}_{i}\left(\mathbf{G}_{2 i+1}(\mathrm{k}) ; \operatorname{St}_{\mathbf{G}_{2 i+1}}(\mathrm{k} ; R)\right)$.

${ }^{*}$ AP is supported in part by NSF grants DMS-1255350 and DMS-1737434.

${ }^{\dagger} \mathrm{SS}$ is supported in part by NSF grant DMS-1500069. 
Remark 1.2. When $\operatorname{char}(\mathrm{k})=2$, the groups $\mathrm{SO}_{n, n}(\mathrm{k})$ and $\mathrm{SO}_{n, n+1}(\mathrm{k})$ in Theorem 1.1 are to be taken naively as the stabilizers of appropriate quadratic forms (see $\$ 2.1$ below); we ignore the Dickson invariant.

Theorem 1.1 (and its proof) is motivated by the following conjecture of Church-FarbPutman. Recall that Borel-Serre [BoSe] proved that the virtual cohomological dimension of $\mathrm{SL}_{n}(\mathbb{Z})$ is $\left(\begin{array}{l}n \\ 2\end{array}\right)$.

Conjecture $1.3\left(\left[\mathrm{CFaP}\right.\right.$, Conjecture 2]). We have $\mathrm{H}^{\left(\begin{array}{l}n \\ 2\end{array}\right)-i}\left(\mathrm{SL}_{n}(\mathbb{Z}) ; \mathbb{Q}\right)=0$ for $n \geq i+2$.

In other words, the rational cohomology of $\mathrm{SL}_{n}(\mathbb{Z})$ vanishes in codimension $i$ as long as $n$ is sufficiently large. Conjecture 1.3 was proved for $i=0$ by Lee-Szczarba [LSz] and for $i=1$ by Church-Putman [CP]. It is open for $i \geq 2$.

To connect Conjecture 1.3 to Theorem 1.1, recall that Borel-Serre [BoSe] proved that $\mathrm{SL}_{n}(\mathbb{Z})$ satisfies a version of Poincaré-Lefschetz duality called virtual Bieri-Eckmann duality. This duality involves a "dualizing module" that measures the "homology at infinity". In our situation, that dualizing module is the Steinberg representation $\mathrm{St}_{\mathrm{SL}_{n}}(\mathbb{Q} ; \mathbb{Q})$ and we have

$$
\mathrm{H}^{\left(\begin{array}{c}
n \\
2
\end{array}\right)-i}\left(\mathrm{SL}_{n}(\mathbb{Z}) ; \mathbb{Q}\right)=\mathrm{H}_{i}\left(\mathrm{SL}_{n}(\mathbb{Z}) ; \operatorname{St}_{\mathrm{SL}_{n}}(\mathbb{Q} ; \mathbb{Q})\right) .
$$

Conjecture 1.3 is thus equivalent to the following conjecture, which resembles Theorem 1.1 for $\mathbf{G}_{n}=\mathrm{SL}_{n}$.

Conjecture 1.4. We have $\mathrm{H}_{i}\left(\mathrm{SL}_{n}(\mathbb{Z}) ; \operatorname{St}_{\mathrm{SL}_{n}}(\mathbb{Q} ; \mathbb{Q})\right)=0$ for $n \geq i+2$.

Remark 1.5. The proofs by Lee-Szczarba [LSz] and Church-Putman [CP] of special cases of Conjecture 1.3 both start by translating things into the language of Conjecture 1.4.

We now briefly describe our proof of Theorem 1.1. As we will discuss in $\S 3$ below, there is a natural inclusion $\mathrm{St}_{\mathbf{G}_{n-1}}(\mathrm{k} ; R) \rightarrow \mathrm{St}_{\mathbf{G}_{n}}(\mathrm{k} ; R)$. This induces a stabilization map

$$
\mathrm{H}_{i}\left(\mathbf{G}_{n-1}(\mathrm{k}) ; \mathrm{St}_{\mathbf{G}_{n-1}}(\mathrm{k} ; R)\right) \rightarrow \mathrm{H}_{i}\left(\mathbf{G}_{n}(\mathrm{k}) ; \mathrm{St}_{\mathbf{G}_{n}}(\mathrm{k} ; R)\right) \text {. }
$$

We will show in $\S 3$ that to prove that $\mathrm{H}_{i}\left(\mathbf{G}_{n}(\mathrm{k}) ; \mathrm{St}_{\mathbf{G}_{n}}(\mathrm{k} ; R)\right)=0$ for large $n$, it is enough to prove the seemingly weaker assertion that (1.1) is a surjection for large $n$. This idea was first introduced by Church-Farb-Putman $[\mathrm{CFaP}]$ as a strategy for proving Conjecture 1.4. It was also noticed by Ash in unpublished work.

The surjectivity of (1.1) is a weak form of homological stability. There is an enormous literature on homological stability theorems. The basic technique underlying most results in the subject goes back to unpublished work of Quillen. In [Dw], Dwyer used these ideas to prove a twisted homological stability theorem for $\mathrm{GL}_{n}(\mathrm{k})$ with quite general coefficient systems. This work was later generalized by van der Kallen [VdK] and very recently by Randal-Williams-Wahl [RaWiWa], whose results cover all the classical groups in Theorem 1.1. Unfortunately, the Steinberg representation does not satisfy the conditions in any of these known theorems. Indeed, these theorems are general enough that if it did, then this would quickly lead to a proof of Conjecture 1.4. Nevertheless, we are able to use some delicate properties of the Steinberg representation to jury-rig the Quillen machine such that it works to prove that (1.1) is surjective for large $n$. 
Remark 1.6. Homological stability for a sequence of groups and homomorphisms $X_{1} \rightarrow$ $X_{2} \rightarrow \cdots$ states that the induced maps $\mathrm{H}_{i}\left(X_{n}\right) \rightarrow \mathrm{H}_{i}\left(X_{n+1}\right)$ are isomorphisms for $n \gg 0$. Alternatively, we can think of each map as "multiplication by $t$ " and give $\oplus_{n} \mathrm{H}_{i}\left(X_{n}\right)$ the structure of a $R[t]$-module, where $R$ denotes our coefficient ring. At least when $R$ is a field, this isomorphism would be a consequence of finite generation.

In our setting, with homology twisted by the Steinberg representation, one should instead think of this map as "multiplication by $t$ " where $t$ is a generator for the exterior algebra in one variable $R[t] / t^{2}$, so that the groups $\mathrm{H}_{i}$ being 0 for $n \gg 0$ would again be a consequence of finite generation. At least when $\mathrm{k}$ is a finite field of size $q$ and $R$ is the field of complex numbers, this is consistent with the idea that $\mathrm{GL}_{n}\left(\mathbf{F}_{q}\right)$ is a $q$-analogue of the symmetric group and the Steinberg representation is the $q$-analogue of its sign representation, which is made more precise via their connection to symmetric functions, see [Mac, §§I.7, IV.4].

Outline. We begin in $\S 2$ with some background and notation. Next, in $\S 3$ we reduce Theorem 1.1 to an appropriate homological stability theorem. We then prove a key isomorphism in $\S 4$. We prove Theorem 1.1 in $\S 5$. This proof depends on a calculation which we perform in $\S 6$.

Convention regarding the empty set. If $X$ is the empty set and $R$ is a commutative ring, then we define $\widetilde{\mathrm{H}}_{-1}(X ; R)=R$. With this convention, if the semisimple k-rank of $\mathbf{G}$ is 0 , then $\mathrm{St}_{\mathbf{G}}(\mathrm{k} ; R)=R$ with the trivial $\mathbf{G}(\mathrm{k})$-action.

Acknowledgments. The second author would like to thank Thomas Church and Benson Farb for many inspiring conversations concerning Conjectures 1.3 and 1.4.

\section{Background and notation}

This section contains some background information and notation needed in the remainder of the paper. It consists of two subsections: $\$ 2.1$ introduces some distinguished parabolic subgroups, and $\S 2.2$ gives some background about the Steinberg representations.

Throughout this section, $\mathrm{k}$ is a field and $\mathbf{G}_{n}$ is either $\mathrm{GL}_{n}, \mathrm{SL}_{n}, \mathrm{Sp}_{2 n}, \mathrm{SO}_{n, n}$, or $\mathrm{SO}_{n, n+1}$.

\subsection{Parabolic and stabilizer subgroups}

Our proof of Theorem 1.1 depends on a careful study of various subgroups of $\mathbf{G}_{n}(\mathrm{k})$. In this section, we will introduce notation for these subgroups: a certain parabolic subgroup $\mathbf{P G}_{n}^{\ell}(\mathrm{k})$, its unipotent radical $\mathbf{U G}_{n}^{\ell}(\mathrm{k})$, a Levi component $\mathbf{L} \mathbf{G}_{n}^{\ell}(\mathrm{k})$ of $\mathbf{P G}_{n}^{\ell}(\mathrm{k})$, and another subgroup $\mathbf{F G}_{n}^{\ell}(\mathrm{k})$ that lies in $\mathbf{P G}_{n}^{\ell}(\mathrm{k})$ and fixes certain vectors.

General and special linear groups. Assume first that $\mathbf{G}_{n}$ is either $\mathrm{GL}_{n}$ or $\mathrm{SL}_{n}$. The group $\mathbf{G}_{n}(\mathrm{k})$ thus acts on the vector space $\mathrm{k}^{n}$, and the k-parabolic subgroups of $\mathbf{G}_{n}(\mathrm{k})$ are the stabilizers of flags of subspaces of $\mathrm{k}^{n}$. Let $\left(\vec{a}_{1}, \ldots, \vec{a}_{n}\right)$ be the standard basis for $\mathrm{k}^{n}$. For 
$1 \leq \ell \leq n$, the group $\mathbf{P G}_{n}^{\ell}(\mathrm{k})$ is defined to be the $\mathbf{G}_{n}(\mathrm{k})$-stabilizer of the flag

$$
0 \subsetneq\left\langle\vec{a}_{1}, \ldots, \vec{a}_{\ell}\right\rangle .
$$

The group $\mathbf{U G}_{n}^{\ell}(\mathrm{k})$ is the subgroup of $\mathbf{P G}_{n}^{\ell}(\mathrm{k})$ consisting of all $M \in \mathbf{P G}_{n}^{\ell}(\mathrm{k})$ that act as the identity on both

$$
\left\langle\vec{a}_{1}, \ldots, \vec{a}_{\ell}\right\rangle \quad \text { and } \mathrm{k}^{n} /\left\langle\vec{a}_{1}, \ldots, \vec{a}_{\ell}\right\rangle \text {. }
$$

The group $\mathbf{L} \mathbf{G}_{n}^{\ell}(\mathrm{k})$ is defined to be the $\mathbf{P G}_{n}^{\ell}(\mathrm{k})$-stabilizer of the flag

$$
0 \subsetneq\left\langle\vec{a}_{\ell+1}, \ldots, \vec{a}_{n}\right\rangle .
$$

If $\mathbf{G}_{n}=\mathrm{GL}_{n}$ then $\mathbf{L G}_{n}^{\ell}(\mathrm{k})$ is the subgroup $\mathrm{GL}_{\ell}(\mathrm{k}) \times \mathrm{GL}_{n-\ell}(\mathrm{k})$ of $\mathbf{G}_{n}$, while if $\mathbf{G}_{n}=\mathrm{SL}_{n}$ then $\mathbf{L G}_{n}^{\ell}(\mathrm{k})$ is the subgroup of $\mathrm{GL}_{\ell}(\mathrm{k}) \times \mathrm{GL}_{n-\ell}(\mathrm{k})$ consisting of matrices of determinant 1 . Finally, define

$$
\mathbf{F G}_{n}^{\ell}(\mathrm{k})=\left\{M \in \mathbf{G}_{n}(\mathrm{k}) \mid M\left(\vec{a}_{j}\right)=\vec{a}_{j} \text { for } 1 \leq j \leq \ell\right\} .
$$

We thus have $\mathbf{F G}_{n}^{\ell}(\mathrm{k}) \subset \mathbf{P G}_{n}^{\ell}(\mathrm{k})$.

Symplectic groups. Now assume that $\mathbf{G}_{n}=\operatorname{Sp}_{2 n}$. Letting $\omega(\cdot, \cdot)$ be the standard symplectic form on $\mathrm{k}^{2 n}$, the group $\mathbf{G}_{n}(\mathrm{k})$ is the subgroup of $\mathrm{GL}_{n}(\mathrm{k})$ consisting of elements that preserve $\omega(\cdot, \cdot)$. The k-parabolic subgroups of $\mathbf{G}_{n}(\mathrm{k})$ are the $\mathbf{G}_{n}(\mathrm{k})$-stabilizers of flags of isotropic subspaces of $\mathrm{k}^{2 n}$, that is, subspaces on which $\omega(\cdot, \cdot)$ vanishes identically. Let $\left(\vec{a}_{1}, \ldots, \vec{a}_{n}, \vec{b}_{1}, \ldots, \vec{b}_{n}\right)$ be the standard symplectic basis for $\mathrm{k}^{2 n}$, so

$$
\omega\left(\vec{a}_{j}, \vec{a}_{j^{\prime}}\right)=\omega\left(\vec{b}_{j}, \vec{b}_{j^{\prime}}\right)=0 \quad \text { and } \quad \omega\left(\vec{a}_{j}, \vec{b}_{j^{\prime}}\right)=\delta_{j j^{\prime}}
$$

for $1 \leq j, j^{\prime} \leq n$, where $\delta_{j j^{\prime}}$ is the Kronecker delta function. For $1 \leq \ell \leq n$, the group $\mathbf{P G}_{n}^{\ell}(\mathrm{k})$ is defined to be the $\mathbf{G}_{n}(\mathrm{k})$-stabilizer of the isotropic flag

$$
0 \subsetneq\left\langle\vec{a}_{1}, \ldots, \vec{a}_{\ell}\right\rangle .
$$

The group $\mathbf{U G}_{n}^{\ell}(\mathrm{k})$ is the subgroup of $\mathbf{P G}_{n}^{\ell}(\mathrm{k})$ consisting of all $M \in \mathbf{P G}_{n}^{\ell}(\mathrm{k})$ that act as the identity on both

$$
\begin{aligned}
\left\langle\vec{a}_{1}, \ldots, \vec{a}_{\ell}\right\rangle \quad \text { and } \quad \begin{array}{l}
\left\langle\vec{a}_{1}, \ldots, \vec{a}_{\ell}\right\rangle^{\perp} /\left\langle\vec{a}_{1}, \ldots, \vec{a}_{\ell}\right\rangle \\
=
\end{array} \quad\left\langle\vec{a}_{1}, \ldots, \vec{a}_{\ell}, \vec{a}_{\ell+1}, \vec{b}_{\ell+1}, \ldots, \vec{a}_{n}, \vec{b}_{n}\right\rangle /\left\langle\vec{a}_{1}, \ldots, \vec{a}_{\ell}\right\rangle .
\end{aligned}
$$

The group $\mathbf{L G}_{n}^{\ell}(\mathrm{k})$ is defined to be the $\mathbf{P G}_{n}^{\ell}(\mathrm{k})$-stabilizer of the isotropic flag

$$
0 \subsetneq\left\langle\vec{b}_{1}, \ldots, \vec{b}_{\ell}\right\rangle .
$$

The group $\mathbf{L G}_{n}^{\ell}(\mathrm{k})$ is thus isomorphic to $\mathrm{GL}_{\ell}(\mathrm{k}) \times \mathbf{G}_{n-\ell}(\mathrm{k})$. Finally, define

$$
\mathbf{F G}_{n}^{\ell}(\mathrm{k})=\left\{M \in \mathbf{G}_{n}(\mathrm{k}) \mid M\left(\vec{a}_{j}\right)=\vec{a}_{j} \text { for } 1 \leq j \leq \ell\right\} .
$$

We thus have $\mathbf{F G}_{n}^{\ell}(\mathrm{k}) \subset \mathbf{P G}_{n}^{\ell}(\mathrm{k})$. 
Orthogonal groups. Finally, assume that $\mathbf{G}_{n}$ is either $\mathrm{SO}_{n, n}$ or $\mathrm{SO}_{n, n+1}$. For an appropriate $m$, the group $\mathbf{G}_{n}(\mathrm{k})$ is then the subgroup of $\mathrm{SL}_{m}(\mathrm{k})$ consisting of elements that preserve a quadratic form $q(\cdot)$ on $\mathrm{k}^{m}$ :

- If $\mathbf{G}_{n}=\mathrm{SO}_{n, n}$, then let $m=2 n$ and let $\left(\vec{a}_{1}, \ldots, \vec{a}_{n}, \vec{b}_{1}, \ldots, \vec{b}_{n}\right)$ be the standard basis for $\mathrm{k}^{m}$. The group $\mathbf{G}_{n}(\mathrm{k})$ is the $\mathrm{SL}_{m}(\mathrm{k})$-stabilizer of the quadratic form $q(\cdot)$ on $\mathrm{k}^{m}$ defined via the formula

$$
q\left(\sum_{j=1}^{n}\left(c_{j} \vec{a}_{j}+d_{j} \vec{b}_{j}\right)\right)=\sum_{j=1}^{n} c_{j} d_{j} \quad\left(c_{j}, d_{j} \in \mathrm{k}\right) .
$$

- If $\mathbf{G}_{n}=\mathrm{SO}_{n, n+1}$, then let $m=2 n+1$ and let $\left(\vec{a}_{1}, \ldots, \vec{a}_{n}, \vec{b}_{1}, \ldots, \vec{b}_{n}, \vec{e}\right)$ be the standard basis for $\mathrm{k}^{m}$. The group $\mathbf{G}_{n}(\mathrm{k})$ is the $\mathrm{SL}_{m}(\mathrm{k})$-stabilizer of the quadratic form $q(\cdot)$ on $\mathrm{k}^{m}$ defined via the formula

$$
q\left(\lambda \vec{e}+\sum_{j=1}^{n}\left(c_{j} \vec{a}_{j}+d_{j} \vec{b}_{j}\right)\right)=\lambda^{2}+\sum_{j=1}^{n} c_{j} d_{j} \quad\left(c_{j}, d_{j}, \lambda \in \mathrm{k}\right) .
$$

In both cases, the k-parabolic subgroups of $\mathbf{G}_{n}(\mathrm{k})$ are the $\mathbf{G}_{n}(\mathrm{k})$-stabilizers of flags of isotropic subspaces of $\mathrm{k}^{m}$, that is, subspaces on which $q(\cdot)$ vanishes identically. For $1 \leq \ell \leq n$ the group $\mathbf{P G}_{n}^{\ell}(\mathrm{k})$ is defined to be the $\mathbf{G}_{n}(\mathrm{k})$-stabilizer of the isotropic flag

$$
0 \subsetneq\left\langle\vec{a}_{1}, \ldots, \vec{a}_{\ell}\right\rangle .
$$

For $\mathbf{G}_{n}=\mathrm{SO}_{n, n}$, the group $\mathbf{U G}_{n}^{\ell}(\mathrm{k})$ is the subgroup of $\mathbf{P} \mathbf{G}_{n}^{\ell}(\mathrm{k})$ consisting of all $M \in \mathbf{P} \mathbf{G}_{n}^{\ell}(\mathrm{k})$ that act as the identity on both

$$
\begin{aligned}
& \left\langle\vec{a}_{1}, \ldots, \vec{a}_{\ell}\right\rangle \quad \text { and } \quad\left\langle\vec{a}_{1}, \ldots, \vec{a}_{\ell}\right\rangle^{\perp} /\left\langle\vec{a}_{1}, \ldots, \vec{a}_{\ell}\right\rangle \\
& =\left\langle\vec{a}_{1}, \ldots, \vec{a}_{\ell}, \vec{a}_{\ell+1}, \vec{b}_{\ell+1}, \ldots, \vec{a}_{n}, \vec{b}_{n}\right\rangle /\left\langle\vec{a}_{1}, \ldots, \vec{a}_{\ell}\right\rangle,
\end{aligned}
$$

while if $\mathbf{G}_{n}=\mathrm{SO}_{n, n+1}$, then the group $\mathbf{U G}_{n}^{\ell}(\mathrm{k})$ is the subgroup of $\mathbf{P} \mathbf{G}_{n}^{\ell}(\mathrm{k})$ consisting of all $M \in \mathbf{P G}_{n}^{\ell}(\mathrm{k})$ that act as the identity on both

$$
\begin{aligned}
& \left\langle\vec{a}_{1}, \ldots, \vec{a}_{\ell}\right\rangle \quad \text { and } \quad\left\langle\vec{a}_{1}, \ldots, \vec{a}_{\ell}\right\rangle^{\perp} /\left\langle\vec{a}_{1}, \ldots, \vec{a}_{\ell}\right\rangle \\
& \quad=\left\langle\vec{a}_{1}, \ldots, \vec{a}_{\ell}, \vec{a}_{\ell+1}, \vec{b}_{\ell+1}, \ldots, \vec{a}_{n}, \vec{b}_{n}, \vec{e}\right\rangle /\left\langle\vec{a}_{1}, \ldots, \vec{a}_{\ell}\right\rangle .
\end{aligned}
$$

The group $\mathbf{L} \mathbf{G}_{n}^{\ell}(\mathrm{k})$ is defined to be the $\mathbf{P G}_{n}^{\ell}(\mathrm{k})$-stabilizer of the isotropic flag

$$
0 \subsetneq\left\langle\vec{b}_{1}, \ldots, \vec{b}_{\ell}\right\rangle
$$

The group $\mathbf{L G}_{n}^{\ell}(\mathrm{k})$ is thus isomorphic to $\mathrm{GL}_{\ell}(\mathrm{k}) \times \mathbf{G}_{n-\ell}(\mathrm{k})$. Finally, define

$$
\mathbf{F G}_{n}^{\ell}(\mathrm{k})=\left\{M \in \mathbf{G}_{n}(\mathrm{k}) \mid M\left(\vec{a}_{j}\right)=\vec{a}_{j} \text { for } 1 \leq j \leq \ell\right\}
$$

We thus have $\mathbf{F G}_{n}^{\ell}(\mathrm{k}) \subset \mathbf{P G}_{n}^{\ell}(\mathrm{k})$. 


\subsection{Facts about the Steinberg representation}

Let $R$ be a commutative ring. The following theorem of Reeder [Re] will play an important role in our proof of Theorem 1.1.

Theorem 2.1 ([Re, Proposition 1.1]). Let $\mathbf{G}$ be a connected reductive group defined over a field $\mathrm{k}$, let $\mathbf{P G}(\mathrm{k})$ be a $\mathrm{k}$-parabolic subgroup of $\mathbf{G}(\mathrm{k})$, and let $\mathbf{L G}(\mathrm{k})$ be a Levi component of $\mathbf{P G}(\mathrm{k})$. Then there exists an $\mathbf{L G}(\mathrm{k})$-equivariant map

$$
\operatorname{St}_{\mathbf{L G}}(\mathrm{k} ; R) \longrightarrow \operatorname{Res}_{\mathbf{L G}(\mathrm{k})}^{\mathrm{G}(\mathrm{k})} \operatorname{St}_{\mathbf{G}}(\mathrm{k} ; R)
$$

such that the induced map

$$
\operatorname{Ind}_{\mathbf{L G}(\mathrm{k})}^{\mathbf{P G}(\mathrm{k})} \operatorname{St}_{\mathbf{L G}}(\mathrm{k} ; R) \rightarrow \operatorname{Res}_{\mathbf{P G}(\mathrm{k})}^{\mathbf{G}(\mathrm{k})} \operatorname{St}_{\mathbf{G}}(\mathrm{k} ; R)
$$

is an isomorphism.

Remark 2.2. The map in Theorem 2.1 is not unique; for instance, it can be post-composed with any element of the unipotent radical of $\mathbf{P G}(\mathrm{k})$. The paper [Re] contains a specific construction of this map, and whenever we refer to the map in Theorem 2.1 we mean the one constructed in [Re].

We wish to apply this to the distinguished parabolic subgroups $\mathbf{P G}_{n}^{\ell}(\mathrm{k})$ that we introduced in $\S 2.1$. To do this, we need to identify $\mathrm{St}_{\mathbf{L G}_{n}^{\ell}}(\mathrm{k} ; R)$.

Lemma 2.3. Let $\mathrm{G}_{n}$ be either $\mathrm{GL}_{n}, \mathrm{SL}_{n}, \mathrm{Sp}_{2 n}, \mathrm{SO}_{n, n}$, or $\mathrm{SO}_{n, n+1}$. Then for all fields $\mathrm{k}$ and all commutative rings $R$, we have

$$
\mathrm{St}_{\mathbf{L G}_{n}^{\ell}}(\mathrm{k} ; R)=\mathrm{St}_{\mathrm{GL}_{\ell}}(\mathrm{k} ; R) \otimes \mathrm{St}_{\mathbf{G}_{n-\ell}}(\mathrm{k} ; R)
$$

for $1 \leq \ell \leq n$.

Proof. For $\mathbf{G}_{n} \neq \mathrm{SL}_{n}$, this follows from the decomposition $\mathbf{L G}_{n}^{\ell}(\mathrm{k})=\mathrm{GL}_{\ell}(\mathrm{k}) \times \mathbf{G}_{n-\ell}(\mathrm{k})$. For $\mathbf{G}_{n}=\mathrm{SL}_{n}$, we instead have that $\mathbf{L G}_{n}^{\ell}(\mathrm{k})$ is the subgroup of $\mathrm{GL}_{\ell}(\mathrm{k}) \times \mathrm{GL}_{n-\ell}(\mathrm{k})$ consisting of matrices of determinant 1 . The lemma in this case follows from two facts:

- There is a bijection between k-parabolic subgroups of $\mathrm{GL}_{n-\ell}(\mathrm{k})$ and $\mathrm{SL}_{n-\ell}(\mathrm{k})$, and thus an $\mathrm{SL}_{n-\ell}(\mathrm{k})$-equivariant isomorphism between $\mathrm{St}_{\mathrm{GL}_{n-\ell}}(\mathrm{k} ; R)$ and $\mathrm{St}_{\mathrm{SL}_{n-\ell}}(\mathrm{k} ; R)$.

- There is a bijection between k-parabolic subgroups of $\mathrm{GL}_{\ell}(\mathrm{k}) \times \mathrm{GL}_{n-\ell}(\mathrm{k})$ and $\mathbf{L G}_{n}^{\ell}(\mathrm{k})$, and thus an $\mathbf{L G}_{n}^{\ell}(\mathrm{k})$-equivariant isomorphism between $\mathrm{St}_{\mathrm{GL}_{\ell}}(\mathrm{k} ; R) \otimes \mathrm{St}_{\mathrm{GL}_{n-\ell}}(\mathrm{k} ; R)$ and $\mathrm{St}_{\mathbf{L} \mathbf{G}_{n}^{\ell}}(\mathrm{k} ; R)$.

Both of these bijections come from taking intersections.

These two results allow us to make the following definition.

Definition 2.4. Let $\mathrm{G}_{n}$ be either $\mathrm{GL}_{n}, \mathrm{SL}_{n}, \mathrm{Sp}_{2 n}, \mathrm{SO}_{n, n}$, or $\mathrm{SO}_{n, n+1}$. Also, let k be a field and $R$ be a commutative ring. For $1 \leq \ell \leq n$, the Reeder product map is the map

$$
\mathrm{St}_{\mathrm{GL}_{\ell}}(\mathrm{k} ; R) \otimes \mathrm{St}_{\mathbf{G}_{n-\ell}}(\mathrm{k} ; R) \longrightarrow \mathrm{St}_{\mathbf{G}_{n}}(\mathrm{k} ; R)
$$

obtained by combining Lemma 2.3 and Theorem 2.1. 
Remark 2.5. Identifying $\operatorname{St}_{\mathrm{GL}_{\ell}}(\mathrm{k} ; R) \otimes \operatorname{St}_{\mathbf{G}_{n-\ell}}(\mathrm{k} ; R)$ with its image in $\operatorname{St}_{\mathbf{G}_{n}}(\mathrm{k} ; R)$ under the Reeder product map, one way of viewing Theorem 2.1 is that it asserts that

$$
\mathrm{St}_{\mathbf{G}_{n}}(\mathrm{k} ; R)=\bigoplus_{u \in \mathbf{U G}_{n}^{\ell}(\mathrm{k})} u \cdot\left(\mathrm{St}_{\mathrm{GL}_{\ell}}(\mathrm{k} ; R) \otimes \mathrm{St}_{\mathbf{G}_{n-\ell}}(\mathrm{k} ; R)\right) .
$$

We will also need the following lemma, which is precisely the case $i=0$ of Theorem 1.1. It generalizes [LSz, Theorem 4.1]. Recall that if $G$ is a group and $M$ is a $G$-module, then the coinvariants $M_{G}$ are the largest quotient of $M$ on which $G$ acts trivially. The coinvariants $M_{G}$ are isomorphic to $\mathrm{H}_{0}(G ; M)$.

Lemma 2.6. Let $\mathrm{G}_{n}$ be either $\mathrm{GL}_{n}, \mathrm{SL}_{n}, \mathrm{Sp}_{2 n}, \mathrm{SO}_{n, n}$, or $\mathrm{SO}_{n, n+1}$. Then for all fields $\mathrm{k}$ and all commutative rings $R$, we have $\left(\mathrm{St}_{\mathbf{G}_{n}}(\mathrm{k} ; R)\right)_{\mathbf{G}_{n}(\mathrm{k} ; R)}=0$ for $n \geq 2$.

Proof. Theorem 2.1 implies that

$$
\operatorname{Ind}_{\mathbf{L G}_{n}^{2}(\mathrm{k})}^{\mathbf{P G}_{n}^{2}(\mathrm{k})} \operatorname{St}_{\mathrm{GL}_{2}}(\mathrm{k} ; R) \otimes \mathrm{St}_{\mathbf{G}_{n-2}}(\mathrm{k} ; R) \cong \operatorname{ReS}_{\mathbf{P G}_{n}^{2}(\mathrm{k})}^{\mathbf{G}_{n}(\mathrm{k})} \operatorname{St}_{\mathbf{G}_{n}}(\mathrm{k} ; R) .
$$

It is thus enough to prove that

$$
\left(\mathrm{St}_{\mathrm{GL}_{2}}(\mathrm{k} ; R) \otimes \mathrm{St}_{\mathbf{G}_{n-2}}(\mathrm{k} ; R)\right)_{\mathbf{L G}_{n}^{2}(\mathrm{k})}=0 .
$$

Whatever $\mathbf{G}_{n}$ is, the group $\mathbf{L G}_{n}^{2}(\mathrm{k})$ contains the subgroup $\mathrm{SL}_{2}(\mathrm{k}) \times 1$. It is thus enough to prove that

$$
\left(\mathrm{St}_{\mathrm{GL}_{2}}(\mathrm{k} ; R)\right)_{\mathrm{SL}_{2}(\mathrm{k})}=0 .
$$

This is an easy exercise using the fact that

$$
\mathrm{St}_{\mathrm{GL}_{2}}(\mathrm{k} ; R)=\widetilde{\mathrm{H}}_{0}\left(\mathcal{T}_{2}(\mathrm{k}) ; R\right)=\widetilde{\mathrm{H}}_{0}\left(\mathbb{P}^{1}(\mathrm{k}) ; \mathbb{Q}\right)
$$

where $\mathbb{P}^{1}(\mathrm{k})$ is the projective line over $\mathrm{k}$, regarded as a discrete set of points. For details, see [LSz, Theorem 4.1].

\section{Reduction to stability}

Let $\mathbf{G}_{n}$ be either $\mathrm{GL}_{n}, \mathrm{SL}_{n}, \mathrm{Sp}_{2 n}, \mathrm{SO}_{n, n}$, or $\mathrm{SO}_{n, n+1}$. Let $\mathrm{k}$ be a field and $R$ be a commutative ring. In this section, we reduce Theorem 1.1 to an appropriate homological stability theorem.

Fix some $i \geq 0$ and some $n \geq 2$. The stabilization map for $\mathrm{H}_{i}\left(\mathbf{G}_{n-1}(\mathrm{k}) ; \operatorname{St}_{\mathbf{G}_{n-1}}(\mathrm{k} ; R)\right)$ is the map

$$
\mathrm{H}_{i}\left(\mathbf{G}_{n-1}(\mathrm{k}) ; \mathrm{St}_{\mathbf{G}_{n-1}}(\mathrm{k} ; R)\right) \rightarrow \mathrm{H}_{i}\left(\mathbf{G}_{n}(\mathrm{k}) ; \operatorname{St}_{\mathbf{G}_{n}}(\mathrm{k} ; R)\right)
$$

induced by the following two maps:

- The group homomorphism $\mathbf{G}_{n-1}(\mathrm{k}) \rightarrow \mathbf{G}_{n}(\mathrm{k})$ obtained as follows. The group $\mathbf{L} \mathbf{G}_{n}^{1}(\mathrm{k})$ is a subgroup of $\mathrm{GL}_{1}(\mathrm{k}) \times \mathbf{G}_{n-1}(\mathrm{k})$ that contains the subgroup $1 \times \mathbf{G}_{n-1}(\mathrm{k})$. In fact, $\mathbf{L G}_{n}^{1}(\mathrm{k})=\mathrm{GL}_{1}(\mathrm{k}) \times \mathbf{G}_{n-1}(\mathrm{k})$ except when $\mathbf{G}_{n}=\mathrm{SL}_{n}$. We can thus define a homomorphism $\mathbf{G}_{n-1}(\mathrm{k}) \rightarrow \mathbf{G}_{n}(\mathrm{k})$ via the composition

$$
\mathbf{G}_{n-1}(\mathrm{k})=1 \times \mathbf{G}_{n-1}(\mathrm{k}) \hookrightarrow \mathbf{L G}_{n}^{1}(\mathrm{k}) \hookrightarrow \mathbf{G}_{n}(\mathrm{k}) .
$$


- The map $\operatorname{St}_{\mathbf{G}_{n-1}}(\mathrm{k} ; R) \rightarrow \mathrm{St}_{\mathbf{G}_{n}}(\mathrm{k} ; R)$ that equals the composition

$$
\mathrm{St}_{\mathbf{G}_{n-1}}(\mathrm{k} ; R) \cong R \otimes \mathrm{St}_{\mathbf{G}_{n-1}}(\mathrm{k} ; R) \cong \mathrm{St}_{\mathrm{GL}_{1}}(\mathrm{k} ; R) \otimes \mathrm{St}_{\mathbf{G}_{n-1}}(\mathrm{k} ; R) \rightarrow \operatorname{St}_{\mathbf{G}_{n}}(\mathrm{k} ; R),
$$

where the final arrow is the Reeder product map. Here we are using the convention regarding the empty set discussed at the end of the introduction which implies that $\mathrm{St}_{\mathrm{GL}_{1}}(\mathrm{k} ; R)=R$; this convention is compatible with Theorem 2.1.

The main result of this section is then as follows.

Lemma 3.1. Let $\mathbf{G}_{n}$ be either $\mathrm{GL}_{n}, \mathrm{SL}_{n}, \mathrm{Sp}_{2 n}, \mathrm{SO}_{n, n}$, or $\mathrm{SO}_{n, n+1}$. Let $\mathrm{k}$ be a field and let $R$ be a commutative ring. Assume that the stabilization map (3.1) is a surjection for $n \geq N$. Then $\mathrm{H}_{i}\left(\mathbf{G}_{n}(\mathrm{k}) ; \operatorname{St}_{\mathbf{G}_{n}}(\mathrm{k} ; R)\right)=0$ for $n \geq N+1$.

Proof. Consider $n \geq N+1$. By assumption, the map

$$
\mathrm{H}_{i}\left(\mathbf{G}_{n-2}(\mathrm{k}) ; \operatorname{St}_{\mathbf{G}_{n-2}}(\mathrm{k} ; R)\right) \rightarrow \mathrm{H}_{i}\left(\mathbf{G}_{n}(\mathrm{k}) ; \operatorname{St}_{\mathbf{G}_{n}}(\mathrm{k} ; R)\right)
$$

obtained by iterating the stabilization map twice is surjective. It is thus enough to show that the image of this map is 0 . We can factor this map as

$$
\begin{aligned}
\mathrm{H}_{i}\left(\mathbf{G}_{n-2}(\mathrm{k}) ; \mathrm{St}_{\mathbf{G}_{n-2}}(\mathrm{k} ; R)\right) & \rightarrow \mathrm{H}_{i}\left(\mathbf{G}_{n-2}(\mathrm{k}) ; \operatorname{St}_{\mathrm{GL}_{2}}(\mathrm{k} ; R) \otimes \mathrm{St}_{\mathbf{G}_{n-2}}(\mathrm{k} ; R)\right) \\
& \rightarrow \mathrm{H}_{i}\left(\mathbf{G}_{n}(\mathrm{k}) ; \operatorname{St}_{\mathbf{G}_{n}}(\mathrm{k} ; R)\right) .
\end{aligned}
$$

Regard $\mathrm{SL}_{2}(\mathrm{k})$ as a subgroup of $\mathbf{G}_{n}(\mathrm{k})$ via the composition

$$
\mathrm{SL}_{2}(\mathrm{k})=\mathrm{SL}_{2}(\mathrm{k}) \times 1 \hookrightarrow \mathbf{L G}_{n}^{2}(\mathrm{k}) \hookrightarrow \mathbf{G}_{n}(\mathrm{k}) .
$$

The subgroup $\mathrm{SL}_{2}(\mathrm{k})$ of $\mathbf{G}_{n}(\mathrm{k})$ commutes with the image of $\mathbf{G}_{n-2}(\mathrm{k})$ in $\mathbf{G}_{n}(\mathrm{k})$ under the map used to define (3.2). Inner automorphisms act trivially on homology, even with twisted coefficients; see [Br2, Proposition III.8.1]. It follows that to show that the image of (3.2) is 0 , it is enough to prove that

$$
\left(\mathrm{St}_{\mathrm{GL}_{2}}(\mathrm{k} ; R) \otimes \mathrm{St}_{\mathbf{G}_{n-2}}(\mathrm{k} ; R)\right)_{\mathrm{SL}_{2}(\mathrm{k})}=0
$$

This is equivalent to

$$
\left(\mathrm{St}_{\mathrm{GL}_{2}}(\mathrm{k} ; R)\right)_{\mathrm{SL}_{2}(\mathrm{k})}=0
$$

which is one case of Lemma 2.6.

\section{The stabilizer subgroups}

This section constructs an isomorphism (Lemma 4.1 below) that will play a fundamental role in our proof of Theorem 1.1.

Let $\mathrm{G}_{n}$ be either $\mathrm{GL}_{n}, \mathrm{SL}_{n}, \mathrm{Sp}_{2 n}, \mathrm{SO}_{n, n}$, or $\mathrm{SO}_{n, n+1}$. Let $\mathrm{k}$ be a field and $R$ be a commutative ring. Fix some $1 \leq \ell \leq n$. There is a map

$$
\mathrm{H}_{i}\left(1 \times \mathbf{G}_{n-\ell}(\mathrm{k}) ; \operatorname{St}_{\mathrm{GL}_{\ell}}(\mathrm{k} ; R) \otimes \operatorname{St}_{\mathbf{G}_{n-\ell}}(\mathrm{k} ; R)\right) \rightarrow \mathrm{H}_{i}\left(\mathbf{F G}_{n}^{\ell}(\mathrm{k}) ; \operatorname{Res}_{\mathbf{F G}_{n}^{\ell}(\mathrm{k})}^{\mathbf{G}_{n}(\mathrm{k})} \operatorname{St}_{\mathbf{G}_{n}}(\mathrm{k} ; R)\right)
$$

induced by the following two maps: 
- The inclusion map $1 \times \mathbf{G}_{n-\ell}(\mathrm{k}) \rightarrow \mathbf{F G}_{n}^{\ell}(\mathrm{k})$. Here we are regarding $\mathbf{F G}_{n}^{\ell}(\mathrm{k})$ as a subgroup of $\mathbf{P G}_{n}^{\ell}(\mathrm{k})$ that contains $1 \times \mathbf{G}_{n-\ell}(\mathrm{k}) \subset \mathbf{L G}_{n}^{\ell}(\mathrm{k}) \subset \mathbf{P G}_{n}^{\ell}(\mathrm{k})$.

- The Reeder product map $\operatorname{St}_{\mathrm{GL}_{\ell}}(\mathrm{k} ; R) \otimes \mathrm{St}_{\mathbf{G}_{n-\ell}}(\mathrm{k} ; R) \rightarrow \mathrm{St}_{\mathbf{G}_{n}}(\mathrm{k} ; R)$.

Our main result is as follows.

Lemma 4.1. Let $\mathrm{k}$ be a field, let $R$ be a commutative ring, let $1 \leq \ell \leq n$, and let $i \geq 0$. Then the map (4.1) is an isomorphism.

Proof. Shapiro's Lemma [Br2, Proposition III.6.2] gives an isomorphism

$$
\begin{aligned}
& \mathrm{H}_{i}\left(1 \times \mathbf{G}_{n-\ell}(\mathrm{k}) ; \operatorname{St}_{\mathrm{GL}_{\ell}}(\mathrm{k} ; R) \otimes \operatorname{St}_{\mathbf{G}_{n-\ell}}(\mathrm{k} ; R)\right) \\
& \quad \cong \mathrm{H}_{i}\left(\mathbf{F G}_{n}^{\ell}(\mathrm{k}) ; \operatorname{Ind}_{1 \times \mathbf{G}_{n-\ell}(\mathrm{k})}^{\mathbf{F G}_{\ell}^{\ell}(\mathrm{k})} \operatorname{St}_{\mathrm{GL}_{\ell}}(\mathrm{k} ; R) \otimes \operatorname{St}_{\mathbf{G}_{n-\ell}}(\mathrm{k} ; R)\right) .
\end{aligned}
$$

Below we will prove that there is an isomorphism

$$
\operatorname{Ind}_{1 \times \mathbf{G}_{n-\ell}(\mathrm{k})}^{\mathbf{F G}_{n}^{\ell}(\mathrm{k})} \operatorname{St}_{\mathrm{GL}_{\ell}}(\mathrm{k} ; R) \otimes \operatorname{St}_{\mathbf{G}_{n-\ell}}(\mathrm{k} ; R) \cong \operatorname{Res}_{\mathbf{F G}_{n}^{\ell}(\mathrm{k})}^{\mathbf{G}_{n}(\mathrm{k})} \operatorname{St}_{\mathbf{G}_{n}}(\mathrm{k} ; R) .
$$

of $\mathbf{F G}_{n}^{\ell}(\mathrm{k})$-representations. Combined with the above, this will yield an isomorphism between the left and right hand sides of (4.1) which is easily seen to be the map in (4.1).

It remains to construct the isomorphism (4.2). Since $\mathbf{F G}_{n}^{\ell}(\mathrm{k}) \subset \mathbf{P G}_{n}^{\ell}(\mathrm{k})$, we can restrict the isomorphism given by Theorem 2.1 and Lemma 2.3 to obtain an isomorphism

$$
\operatorname{Res}_{\mathbf{F G}_{n}^{\ell}(\mathrm{k})}^{\mathbf{P G}_{\ell}^{\ell}(\mathrm{k})} \operatorname{Ind}_{\mathbf{L G}_{n}^{\ell}(\mathrm{k})}^{\mathbf{P G}_{\ell}^{\ell}(\mathrm{k})} \operatorname{St}_{\mathrm{GL}_{\ell}}(\mathrm{k} ; R) \otimes \operatorname{St}_{\mathbf{G}_{n-\ell}}(\mathrm{k} ; R) \cong \operatorname{Res}_{\mathbf{F G}_{n}^{\ell}(\mathrm{k})}^{\mathbf{G}_{n}(\mathrm{k})} \operatorname{St}_{\mathbf{G}_{n}}(\mathrm{k} ; R) .
$$

The unipotent radical of $\mathbf{P G}_{n}^{\ell}(\mathrm{k})$ is contained in $\mathbf{F G}_{n}^{\ell}(\mathrm{k})$. This implies that there is a single $\left(\mathbf{F G}_{n}^{\ell}(\mathrm{k}), \mathbf{L} \mathbf{G}_{n}^{\ell}(\mathrm{k})\right)$-double coset in $\mathbf{P G}_{n}^{\ell}(\mathrm{k})$. Also, $\mathbf{F G}_{n}^{\ell}(\mathrm{k}) \cap \mathbf{L} \mathbf{G}_{n}^{\ell}(\mathrm{k})=\mathbf{G}_{n-\ell}(\mathrm{k})$. The double coset formula [Br2, Proposition III.5.6b] therefore implies that the left side of (4.3) is canonically isomorphic to

$\operatorname{Ind}_{1 \times \mathbf{G}_{n-\ell}(\mathrm{k})}^{\mathbf{F G}_{\ell}^{\ell}(\mathrm{k})} \operatorname{Res}_{1 \times \mathbf{G}_{n-\ell}(\mathrm{k})}^{\mathbf{L G} \mathbf{G}_{\ell}^{\ell}(\mathrm{k})} \operatorname{St}_{\mathrm{GL}_{\ell}}(\mathrm{k} ; R) \otimes \operatorname{St}_{\mathbf{G}_{n-\ell}}(\mathrm{k} ; R)=\operatorname{Ind}_{1 \times \mathbf{G}_{n-\ell}(\mathrm{k})}^{\mathbf{F G}_{n}^{\ell}(\mathrm{k})} \operatorname{St}_{\mathrm{GL}_{\ell}}(\mathrm{k} ; R) \otimes \operatorname{St}_{\mathbf{G}_{n-\ell}}(\mathrm{k} ; R)$, as desired.

The following alternate version of Lemma 4.1 will be useful.

Corollary 4.2. Let $\mathrm{k}$ be a field, let $R$ be a commutative ring, let $1 \leq \ell \leq n$, and let $i \geq 0$. Then there exists an isomorphism

$$
\operatorname{St}_{\mathrm{GL}_{\ell}}(\mathrm{k} ; R) \otimes \mathrm{H}_{i}\left(\mathbf{G}_{n-\ell}(\mathrm{k}) ; \operatorname{St}_{\mathbf{G}_{n-\ell}}(\mathrm{k} ; R)\right) \cong \mathrm{H}_{i}\left(\mathbf{F G}_{n}^{\ell}(\mathrm{k}) ; \operatorname{Res}_{\mathbf{F G}_{n}^{\ell}(\mathrm{k})}^{\mathbf{G}_{n}(\mathrm{k})} \operatorname{St}_{\mathbf{G}_{n}}(\mathrm{k} ; R)\right) .
$$

Proof. Since $\mathrm{St}_{\mathrm{GL}}(\mathrm{k} ; R)$ is a free $R$-module, we have

$$
\mathrm{St}_{\mathrm{GL} \ell}(\mathrm{k} ; R) \otimes \mathrm{H}_{i}\left(\mathbf{G}_{n-\ell}(\mathrm{k}) ; \operatorname{St}_{\mathbf{G}_{n-\ell}}(\mathrm{k} ; R)\right) \cong \mathrm{H}_{i}\left(1 \times \mathbf{G}_{n-\ell}(\mathrm{k}) ; \operatorname{St}_{\mathrm{GL}_{\ell}}(\mathrm{k} ; R) \otimes \operatorname{St}_{\mathbf{G}_{n-\ell}}(\mathrm{k} ; R)\right) .
$$

The corollary now follows from Lemma 4.1.

We will also need an explicit inverse

$$
\mathrm{H}_{i}\left(\mathbf{F G}_{n}^{\ell}(\mathrm{k}) ; \operatorname{Res}_{\mathbf{F G}_{n}^{\ell}(\mathrm{k})}^{\mathbf{G}_{n}(\mathrm{k})} \operatorname{St}_{\mathbf{G}_{n}}(\mathrm{k} ; R)\right) \rightarrow \mathrm{H}_{i}\left(1 \times \mathbf{G}_{n-\ell}(\mathrm{k}) ; \operatorname{St}_{\mathrm{GL}_{\ell}}(\mathrm{k} ; R) \otimes \operatorname{St}_{\mathbf{G}_{n-\ell}}(\mathrm{k} ; R)\right)
$$

to the isomorphism (4.1). The map (4.4) will be induced by the following two maps: 
- The homomorphism $\mathbf{F G}_{n}^{\ell}(\mathrm{k}) \rightarrow 1 \times \mathbf{G}_{n-\ell}(\mathrm{k})$ obtained by restricting the projection $\mathbf{P G}_{n}^{\ell}(\mathrm{k}) \rightarrow \mathbf{L G}_{n}^{\ell}(\mathrm{k})$ to $\mathbf{F G}_{n}^{\ell}(\mathrm{k})$.

- The map

$$
\mathrm{St}_{\mathbf{G}_{n}}(\mathrm{k} ; R) \rightarrow \mathrm{St}_{\mathrm{GL}_{\ell}}(\mathrm{k} ; R) \otimes \mathrm{St}_{\mathbf{G}_{n-\ell}}(\mathrm{k} ; R)
$$

which equals the composition

$$
\begin{aligned}
\operatorname{St}_{\mathbf{G}_{n}}(\mathrm{k} ; R) & =\bigoplus_{u \in \mathbf{U G}_{n}^{\ell}(\mathrm{k})} u \cdot\left(\operatorname{St}_{\mathrm{GL}_{\ell}}(\mathrm{k} ; R) \otimes \operatorname{St}_{\mathbf{G}_{n-\ell}}(\mathrm{k} ; R)\right) \\
& \rightarrow \operatorname{St}_{\mathrm{GL}_{\ell}}(\mathrm{k} ; R) \otimes \operatorname{St}_{\mathbf{G}_{n-\ell}}(\mathrm{k} ; R),
\end{aligned}
$$

where the first equality comes from a combination of Theorem 2.1 and Lemma 2.3 (see Remark 2.5) and the last arrow takes $u \cdot x \in u \cdot\left(\operatorname{St}_{\mathrm{GL}_{\ell}}(\mathrm{k} ; R) \otimes \operatorname{St}_{\mathbf{G}_{n-\ell}}(\mathrm{k} ; R)\right)$ to $x \in \mathrm{St}_{\mathrm{GL}_{\ell}}(\mathrm{k} ; R) \otimes \mathrm{St}_{\mathbf{G}_{n-\ell}}(\mathrm{k} ; R)$. We will call this map the Reeder projection map.

It is clear that these define a map of the form (4.4). The following lemma says that this is an inverse to (4.1).

Lemma 4.3. Let $\mathrm{k}$ be a field, let $R$ be a commutative ring, let $1 \leq \ell \leq n$, and let $i \geq 0$. Then the map (4.4) is an inverse to the map (4.1).

Proof. Immediate from the fact that the compositions

$$
1 \times \mathbf{G}_{n-\ell}(\mathrm{k}) \rightarrow \mathbf{F G}_{n}^{\ell}(\mathrm{k}) \rightarrow 1 \times \mathbf{G}_{n-\ell}(\mathrm{k})
$$

and

$$
\mathrm{St}_{\mathrm{GL}}(\mathrm{k} ; R) \otimes \mathrm{St}_{\mathbf{G}_{n-\ell}}(\mathrm{k} ; R) \rightarrow \mathrm{St}_{\mathbf{G}_{n}}(\mathrm{k} ; R) \rightarrow \mathrm{St}_{\mathrm{GL}_{\ell}}(\mathrm{k} ; R) \otimes \operatorname{St}_{\mathbf{G}_{n-\ell}}(\mathrm{k} ; R)
$$

of the maps used to define (4.1) and (4.4) equal the identity.

\section{$5 \quad$ Vanishing}

This section is devoted to the proof of Theorem 1.1. The actual proof is in $§ 5.3$. This is preceded by two sections of preliminaries.

\subsection{Equivariant homology}

In our proof of Theorem 1.1, we will need some basic facts about equivariant homology. A basic reference is [Br2, Chapter VII.7].

Let $G$ be a group, let $X$ be a semisimplicial set on which $G$ acts, let $R$ be a ring, and let $M$ be an $R[G]$-module. Let $E G$ be a contractible semisimplicial set on which $G$ acts freely and let $B G=E G / G$, so $B G$ is a classifying space for $G$. Denote by $E G \times_{G} X$ the quotient of $E G \times X$ by the diagonal action of $G$. This is known as the Borel construction. The homotopy type of $E G \times_{G} X$ does not depend on the choice of $E G$. The projection $E G \times_{G} X \rightarrow E G / G=B G$ induces a homomorphism $\pi_{1}\left(E G \times_{G} X\right) \rightarrow \pi_{1}(B G)=G$. Via this homomorphism, we can regard $M$ as a local coefficient system on $E G \times_{G} X$. The $G$-equivariant homology groups of $X$ with coefficients in $M$, denoted $\mathrm{H}_{*}^{G}(X ; M)$, are the homology groups of $E G \times_{G} X$ with respect to the local coefficient system $M$. 
Lemma 5.1. If $X$ is $\ell$-connected, then the above map $E G \times_{G} X \rightarrow E G / G=B G$ induces an isomorphism $\mathrm{H}_{i}^{G}(X ; M) \cong \mathrm{H}_{i}(G ; M)$ for $0 \leq i \leq \ell$ and a surjection $\mathrm{H}_{\ell+1}^{G}(X ; M) \rightarrow$ $\mathrm{H}_{\ell+1}(X ; M)$.

Proof. The group $G$ acts freely on $E G \times X$ and $E G \times X$ is $\ell$-connected. Viewing $E G \times X$ as a CW-complex, we can make $E G \times X$ contractible by adding cells of dimension at least $(\ell+2)$. We conclude that there exists a classifying space for $G$ whose $(\ell+1)$-skeleton equals the $(\ell+1)$-skeleton of $E G \times_{G} X$. The lemma follows.

Our main tool for understanding $\mathrm{H}_{*}^{G}(X ; M)$ is the following spectral sequence, which is constructed in [Br2, Equation VII.7.7].

Lemma 5.2. For all $p \geq 0$, let $\Sigma_{p}$ be a set containing exactly one representative for each orbit of the action of $G$ on the p-simplices of $X$. For $\sigma \in \Sigma_{p}$, let $G_{\sigma}$ be the stabilizer of $\sigma$. Then there is a first quadrant spectral sequence

$$
E_{p, q}^{1}=\bigoplus_{\sigma \in \Sigma_{p}} \mathrm{H}_{q}\left(G_{\sigma} ; \operatorname{Res}_{G_{\sigma}}^{G} M\right) \Longrightarrow \mathrm{H}_{p+q}^{G}(X ; M)
$$

Remark 5.3. In [Br2, Equation VII.7.7], the action of $G_{\sigma}$ on $M$ is twisted by an "orientation character"; however, this is unnecessary in our situation, since we are working with semisimplicial sets rather than ordinary simplicial complexes (the point being that in the geometric realization, the setwise stabilizer of a simplex stabilizes the simplex pointwise).

\subsection{Complexes of partial bases}

Let $\mathrm{k}$ be a field and let $\mathrm{G}_{n}$ be either $\mathrm{GL}_{n}, \mathrm{SL}_{n}, \mathrm{Sp}_{2 n}, \mathrm{SO}_{n, n}$, or $\mathrm{SO}_{n, n+1}$. To prove Theorem 1.1, we will need to construct a highly connected space $\mathbf{C G}_{n}(\mathrm{k})$ on which $\mathbf{G}_{n}(\mathrm{k})$ acts. The definition of this complex is as follows.

- If $\mathbf{G}_{n}=\mathrm{GL}_{n}$ or $\mathbf{G}_{n}=\mathrm{SL}_{n}$, then define $\mathbf{C G}_{n}(\mathrm{k})$ to be the complex of partial bases for $\mathrm{k}^{n}$, i.e. the semisimplicial complex whose $\ell$-simplices are ordered sequences $\left[\vec{v}_{0}, \ldots, \vec{v}_{\ell}\right]$ of linearly independent elements of $\mathrm{k}^{n}$.

- If $\mathbf{G}_{n}=\mathrm{Sp}_{2 n}$ or $\mathbf{G}_{n}=\mathrm{SO}_{n, n}$ or $\mathbf{G}_{n}=\mathrm{SO}_{n, n+1}$ and $\mathrm{k}^{m}$ is the vector space upon which $\mathbf{G}_{n}(\mathrm{k})$ acts (so $m$ is either $2 n$ or $2 n+1$ ), then define $\mathbf{C G}_{n}(\mathrm{k})$ to be the complex of partial isotropic bases for $\mathrm{k}^{m}$, i.e. the semisimplicial complex whose $\ell$-simplices are ordered sequences $\left[\vec{v}_{0}, \ldots, \vec{v}_{\ell}\right]$ of linearly independent elements of $\mathrm{k}^{m}$ that span an isotropic subspace.

The following theorem summarizes the properties of $\mathbf{C G}_{n}(\mathrm{k})$.

Theorem 5.4. Let $\mathrm{k}$ be a field and let $\mathrm{G}_{n}$ be either $\mathrm{GL}_{n}, \mathrm{SL}_{n}, \mathrm{Sp}_{2 n}, \mathrm{SO}_{n, n}$, or $\mathrm{SO}_{n, n+1}$. The following then hold.

1. The group $\mathbf{G}_{n}(\mathrm{k})$ acts transitively on the $\ell$-cells of $\mathbf{C G}_{n}(\mathrm{k})$ for all $0 \leq \ell<n-1$.

2. The space $\mathbf{C G}_{n}(\mathrm{k})$ is $f(n)$-connected where $f(n)$ is given by:

(a) $f(n)=n-2$ if $\mathbf{G}_{n}$ is either $\mathrm{GL}_{n}$ or $\mathrm{SL}_{n}$,

(b) $f(n)=\frac{n-3}{2}$ if $\mathbf{G}_{n}$ is either $\mathrm{Sp}_{2 n}, \mathrm{SO}_{n, n}$, or $\mathrm{SO}_{n, n+1}$. 
Proof. The first assertion is well known (and also holds for $\ell=n-1$ except when $\mathbf{G}_{n}=\mathrm{SL}_{n}$ ). As for the second, Maazen proved in his thesis [Maa] that $\mathbf{C G}_{n}(\mathrm{k})$ is $(n-2)$-connected for $\mathbf{G}_{n}=\mathrm{GL}_{n}$ and $\mathbf{G}_{n}=\mathrm{SL}_{n}$. See $[\mathrm{VdK}]$ for a published proof of a more general result. Friedrich proved in [Fr, Theorem 3.23] that $\mathbf{C G}_{n}(\mathrm{k})$ is $\frac{n-3}{2}$-connected for $\mathbf{G}_{n}=\mathrm{Sp}_{2 n}$ and $\mathbf{G}_{n}=\mathrm{SO}_{n, n}$ and $\mathbf{G}_{n}=\mathrm{SO}_{n, n+1}$ (for $\mathrm{Sp}_{2 n}$ and $\mathrm{SO}_{n, n}$, this was proven earlier in [MiVdK, Theorem 7.3]). To apply the cited result of Friedrich to our situation, we need the fact that the unitary stable rank of a field is 1 (see, e.g., [MiVdK, Example 6.5]).

\subsection{The proof of Theorem 1.1}

Let us first recall the statement of the theorem. Let $\mathbf{G}_{n}$ be either $\mathrm{GL}_{n}, \mathrm{SL}_{n}, \mathrm{Sp}_{2 n}, \mathrm{SO}_{n, n}$, or $\mathrm{SO}_{n, n+1}$. Also, let $\mathrm{k}$ be a field and $R$ be a commutative ring. Our goal is to prove that $\mathrm{H}_{i}\left(\mathbf{G}_{n}(\mathrm{k}) ; \operatorname{St}_{\mathbf{G}_{n}}(\mathrm{k} ; R)\right)=0$ for $n \geq 2 i+2$ and that there exists a surjection

$$
\mathrm{H}_{i}\left(\mathbf{G}_{2 i}(\mathrm{k}) ; \mathrm{St}_{\mathbf{G}_{2 i}}(\mathrm{k} ; R)\right) \rightarrow \mathrm{H}_{i}\left(\mathbf{G}_{2 i+1}(\mathrm{k}) ; \operatorname{St}_{\mathbf{G}_{2 i+1}}(\mathrm{k} ; R)\right) .
$$

Of course, this surjection will be induced by the stabilization map defined in $\S 3$.

The proof is by induction on $i$. We begin with the base case $i=0$. Lemma 2.6 says that $\mathrm{H}_{0}\left(\mathbf{G}_{n}(\mathrm{k}) ; \mathrm{St}_{\mathbf{G}_{n}}(\mathrm{k} ; R)\right)=0$ for $n \geq 2$, so we only need to show that the map (5.1) is a surjection for $i=0$. For the domain, $\mathbf{G}_{0}(\mathrm{k})$ is the trivial group. By our convention regarding the empty set discussed at the end of the introduction, we thus have $\operatorname{St}_{\mathbf{G}_{0}}(\mathrm{k} ; R)=R$, and hence $\mathrm{H}_{i}\left(\mathbf{G}_{0}(\mathrm{k}) ; \mathrm{St}_{\mathbf{G}_{0}}(\mathrm{k} ; R)\right)=R$. To simplify the codomain, we have several cases.

- $\mathrm{G}_{1}=\mathrm{GL}_{1}$ or $\mathbf{G}_{1}=\mathrm{SO}_{1,1}$. In fact, these groups are isomorphic and are commutative, so $\operatorname{St}_{\mathbf{G}_{1}}(\mathrm{k} ; R)=R$ in these cases and (5.1) is an isomorphism.

- $\mathbf{G}_{1}=\mathrm{SL}_{1}$. The group $\mathrm{SL}_{1}$ is the trivial group and thus $\mathrm{St}_{\mathbf{G}_{1}}(\mathrm{k} ; R)=R$ and (5.1) is an isomorphism.

- $\mathrm{G}_{1}=\mathrm{Sp}_{2} \cong \mathrm{SL}_{2}$ or $\mathbf{G}_{1}=\mathrm{SO}_{2,1} \cong \mathrm{PSL}_{2}$. These groups have isomorphic Steinberg representations and the action of $\mathrm{SL}_{2}(\mathrm{k})$ on $\mathrm{St}_{\mathrm{SL}_{2}}(\mathrm{k} ; R)$ factors through $\mathrm{PSL}_{2}(\mathrm{k})$. This case thus follows from Lemma 2.6, which says that $\mathrm{H}_{0}\left(\mathrm{SL}_{2}(\mathrm{k}) ; \mathrm{St}_{\mathrm{SL}_{2}}(\mathrm{k} ; R)\right)=0$.

This completes the base case.

Assume now that $i>0$ and that the desired result is true for all smaller values of $i$. We will prove that the stabilization map

$$
\mathrm{H}_{i}\left(\mathbf{G}_{n-1}(\mathrm{k}) ; \mathrm{St}_{\mathbf{G}_{n-1}}(\mathrm{k} ; R)\right) \rightarrow \mathrm{H}_{i}\left(\mathbf{G}_{n}(\mathrm{k}) ; \operatorname{St}_{\mathbf{G}_{n}}(\mathrm{k} ; R)\right)
$$

is surjective for $n \geq 2 i+1$. Lemma 3.1 will then imply that $\mathrm{H}_{i}\left(\mathbf{G}_{n}(\mathrm{k}) ; \operatorname{St}_{\mathbf{G}_{n}}(\mathrm{k} ; R)\right)=0$ for $n \geq 2 i+2$, and the theorem will follow.

Fix some $n \geq 2 i+1$ and let $\mathrm{k}^{m}$ be the standard vector space representation of $\mathbf{G}_{n}(\mathrm{k}$ ) (so $m$ is either $n, 2 n$, or $2 n+1)$. Let $\left\{\vec{a}_{1}, \ldots, \vec{a}_{n}\right\}$ be the vectors in $\mathrm{k}^{m}$ such that

$$
\mathbf{F G}_{n}^{\ell}(\mathrm{k})=\left\{M \in \mathbf{G}_{n}(\mathrm{k}) \mid M\left(\vec{a}_{j}\right)=\vec{a}_{j} \text { for } 1 \leq j \leq \ell\right\}
$$

for $1 \leq \ell \leq n$. Combining the second conclusion of Theorem 5.4 with Lemma 5.1, we have a surjection

$$
\mathrm{H}_{i}^{\mathbf{G}_{n}(\mathrm{k})}\left(\mathbf{C G}_{n}(\mathrm{k}) ; \mathrm{St}_{\mathbf{G}_{n}}(\mathrm{k} ; R)\right) \rightarrow \mathrm{H}_{i}\left(\mathbf{G}_{n}(\mathrm{k}) ; \operatorname{St}_{\mathbf{G}_{n}}(\mathrm{k} ; R)\right) .
$$

We will analyze $\mathrm{H}_{i}^{\mathbf{G}_{n}(\mathrm{k})}\left(\mathbf{C G}_{n}(\mathrm{k}) ; \mathrm{St}_{\mathbf{G}_{n}}(\mathrm{k} ; R)\right)$ using the spectral sequence from Lemma 5.2. To calculate its $E^{1}$-page, observe that the first conclusion of Theorem 5.4 says that $\mathbf{G}_{n}(\mathrm{k})$ 
acts transitively on the $p$-simplices of $\mathbf{C G}_{n}(\mathrm{k})$ for $0 \leq p<n-1$. The stabilizer of the $(p-1)$-simplex $\left[\vec{a}_{1}, \ldots, \vec{a}_{p}\right]$ is $\mathbf{F G}_{n}^{p}(\mathrm{k})$, so the spectral sequence in Lemma 5.2 has

$$
E_{p, q}^{1}=\mathrm{H}_{q}\left(\mathbf{F G}_{n}^{p+1}(\mathrm{k}) ; \operatorname{Res}_{\mathbf{F G}_{n}^{p+1}(\mathrm{k})}^{\mathbf{G}_{n}(\mathrm{k})} \operatorname{St}_{\mathbf{G}_{n}}(\mathrm{k} ; R)\right),
$$

for $0 \leq p<n-1$.

We will prove that all of the terms on the $p+q=i$ line of the $E^{\infty}$-page of our spectral sequence vanish except for possibly the term $E_{0, i}^{\infty}$. To do this, consider $p, q \geq 0$ with $p+q=i$ and $p \geq 1$. The case $p=1$ and $n=2 i+1$ is exceptional and must be treated separately. To avoid getting bogged down here, we postpone this calculation until $\S 6$ below, where it appears as Lemma 6.1. ${ }^{1}$

We thus can assume that either $p \geq 2$ or that $n \geq 2 i+2$. Since $n \geq 2 i+1$, we certainly have $p<n-1$, so $E_{p, q}^{1}$ is in the regime where the above description of the $E^{1}$-page holds. Applying Corollary 4.2 to (5.4), we see that

$$
E_{p, q}^{1}=\operatorname{St}_{\mathrm{GL}_{p+1}}(\mathrm{k} ; R) \otimes \mathrm{H}_{q}\left(\mathbf{G}_{n-p-1}(\mathrm{k}) ; \operatorname{St}_{\mathbf{G}_{n-p-1}}(\mathrm{k} ; R)\right) .
$$

To see that this vanishes, it is enough to show that $\mathrm{H}_{q}\left(\mathbf{G}_{n-p-1}(\mathrm{k}) ; \operatorname{St}_{\mathbf{G}_{n-p-1}}(\mathrm{k} ; R)\right)=0$. This is a consequence of our inductive hypothesis; to see that it applies, observe that if $p \geq 2$ then

$$
n-p-1 \geq(2 i+1)-p-1=2(p+q)-p=2 q+p \geq 2 q+2,
$$

while if $p=1$ and $n \geq 2 i+2$ then

$$
n-p-1 \geq(2 i+2)-1-1=2(p+q)=2 q+2 .
$$

This implies that $E_{p, q}^{1}=0$, and thus that $E_{p, q}^{\infty}=0$.

The $p+q=i$ line of the $E^{\infty}$-page of our spectral sequence thus only has a single potentially nonzero entry, namely $E_{0, i}^{\infty}$, and this is a quotient of

$$
E_{0, i}^{1}=\mathrm{H}_{i}\left(\mathbf{F G}_{n}^{1}(\mathrm{k}) ; \operatorname{Res}_{\mathbf{F G}_{n}^{1}(\mathrm{k})}^{\mathbf{G}_{n}(\mathrm{k})} \operatorname{St}_{\mathbf{G}_{n}}(\mathrm{k} ; R)\right) .
$$

This entry thus surjects onto $\mathrm{H}_{i}^{\mathbf{G}_{n}(\mathrm{k})}\left(\mathbf{C G}_{n}(\mathrm{k}) ; \mathrm{St}_{\mathbf{G}_{n}}(\mathrm{k} ; R)\right)$. Combining this with the surjection (5.3), we obtain a surjection

$$
\mathrm{H}_{i}\left(\mathbf{F G}_{n}^{1}(\mathrm{k}) ; \operatorname{Res}_{\mathbf{F G}_{n}^{1}(\mathrm{k})}^{\mathbf{G}_{n}(\mathrm{k})} \operatorname{St}_{\mathbf{G}_{n}}(\mathrm{k} ; R)\right) \longrightarrow \mathrm{H}_{i}\left(\mathbf{G}_{n}(\mathrm{k}) ; \mathrm{St}_{\mathbf{G}_{n}}(\mathrm{k} ; R)\right) .
$$

Examining the construction of our spectral sequence in [Br2, Chapter VII.7], it is easy to see that this comes from the map induced by the inclusion $\mathbf{F G}_{n}^{1}(\mathrm{k}) \hookrightarrow \mathbf{G}_{n}(\mathrm{k})$. Combining (5.5) with the isomorphism

$$
\mathrm{H}_{i}\left(\mathbf{G}_{n-1}(\mathrm{k}) ; \operatorname{St}_{\mathbf{G}_{n-1}}(\mathrm{k} ; R)\right) \stackrel{\cong}{\rightrightarrows} \mathrm{H}_{i}\left(\mathbf{F G}_{n}^{1}(\mathrm{k}) ; \operatorname{Res}_{\mathbf{F G}_{n}^{1}(\mathrm{k})}^{\mathbf{G}_{n}(\mathrm{k})} \operatorname{St}_{\mathbf{G}_{n}}(\mathrm{k} ; R)\right)
$$

given by the $\ell=1$ case of Corollary 4.2, we conclude that (5.2) is a surjection, as desired.

\footnotetext{
${ }^{1}$ This exceptional case could be avoided at the cost of only proving that $\mathrm{H}_{i}\left(\mathbf{G}_{n} ; \mathrm{St}_{\mathbf{G}_{n}}(\mathrm{k} ; R)\right)=0$ for $n \geq 3 i+2$ instead of for $n \geq 2 i+2$.
} 


\section{Killing the exceptional term in the spectral sequence}

This section is devoted to proving the vanishing result postponed from the proof of Theorem 1.1 in $\S 5.3$. The notation in this section is thus identical to that in $\S 5.3$ :

- $\mathrm{G}_{n}$ is either $\mathrm{GL}_{n}, \mathrm{SL}_{n}, \mathrm{Sp}_{2 n}, \mathrm{SO}_{n, n}$, or $\mathrm{SO}_{n, n+1}$.

- $\mathrm{k}$ is a field and $R$ is a commutative ring.

- $i>0$ and $n=2 i+1$ (the only case that remained in that section).

- $\mathrm{k}^{m}$ is the standard vector space representation of $\mathbf{G}_{n}(\mathrm{k})$ (so $m$ is either $n, 2 n$, or $2 n+1$ ).

- $\left\{\vec{a}_{1}, \ldots, \vec{a}_{n}\right\}$ is the set of vectors in $\mathrm{k}^{m}$ such that

$$
\mathbf{F G}_{n}^{\ell}(\mathrm{k})=\left\{M \in \mathbf{G}_{n}(\mathrm{k}) \mid M\left(\vec{a}_{j}\right)=\vec{a}_{j} \text { for } 1 \leq j \leq \ell\right\}
$$

for $1 \leq \ell \leq n$.

- $E_{p, q}^{r}$ is the spectral sequence from Lemma 5.2 converging to $\mathrm{H}_{i}^{\mathbf{G}_{n}(\mathrm{k})}\left(\mathbf{C G}_{n}(\mathrm{k}) ; \operatorname{St}_{\mathbf{G}_{n}}(\mathrm{k} ; R)\right)$. What we must prove is as follows.

Lemma 6.1. Let the notation be as above, and assume that the stabilization map

$$
\mathrm{H}_{i-1}\left(\mathbf{G}_{n-3}(\mathrm{k}) ; \operatorname{St}_{\mathbf{G}_{n-3}}(\mathrm{k} ; R)\right) \rightarrow \mathrm{H}_{i-1}\left(\mathbf{G}_{n-2}(\mathrm{k}) ; \operatorname{St}_{\mathbf{G}_{n-2}}(\mathrm{k} ; R)\right)
$$

is surjective. Then the differential $E_{2, i-1}^{1} \rightarrow E_{1, i-1}^{1}$ is surjective, and thus $E_{1, i-1}^{\infty}=0$.

The proof of Lemma 6.1 is divided into five sections:

- In $\$ 6.1$, we give an explicit form for the differential $E_{2, i-1}^{1} \rightarrow E_{1, i-1}^{1}$.

- In $\$ 6.2$, we translate that explicit form into one involving the stabilization map (6.1).

- In $\S 6.3$, we summarize what remains to be proved.

- In $\$ 6.4$, we give some needed background information about apartments.

- In $\S 6.5$, we finish off the proof of Lemma 6.1.

\subsection{Identifying the differential}

The notation is as in the beginning of $\S 6$. In this section, we identify the differential $E_{2, i-1}^{1} \rightarrow$ $E_{1, i-1}^{1}$. Since $n=2 i+1$ and $i>0$, we have $1<n-1$, so $E_{1, i-1}^{1}$ is as described in (5.4), i.e.

$$
E_{1, i-1}^{1} \cong \mathrm{H}_{i-1}\left(\mathbf{F G}_{n}^{2}(\mathrm{k}) ; \operatorname{Res}_{\mathbf{F G}_{n}^{2}(\mathrm{k})}^{\mathbf{G}_{n}(\mathrm{k})} \operatorname{St}_{\mathbf{G}_{n}}(\mathrm{k} ; R)\right)
$$

If $i=1$, then we do not have $2<n-1$, so in this case $E_{2, i-1}^{1}$ is not as described in (5.4). The issue is that $\mathbf{G}_{n}(\mathrm{k})$ might not act transitively on the 2-simplices of $\mathbf{C G}_{n}(\mathrm{k})$ (this is actually only a problem for $\mathbf{G}_{n}=\mathrm{SL}_{n}$ ). However, for all values of $i$ it is still the case that $E_{2, i-1}^{1}$ contains

$$
\mathrm{H}_{i-1}\left(\mathbf{F G}_{n}^{3}(\mathrm{k}) ; \operatorname{Res}_{\mathbf{F G}_{n}^{3}(\mathrm{k})}^{\mathbf{G}_{n}(\mathrm{k})} \operatorname{St}_{\mathbf{G}_{n}}(\mathrm{k} ; R)\right)
$$

as a summand. The restriction of the differential $E_{2, i-1}^{1} \rightarrow E_{1, i-1}^{1}$ to this summand is a map

$$
\partial: \mathrm{H}_{i-1}\left(\mathbf{F G}_{n}^{3}(\mathrm{k}) ; \operatorname{Res}_{\mathbf{F G}_{n}^{3}(\mathrm{k})}^{\mathbf{G}_{n}(\mathrm{k})} \operatorname{St}_{\mathbf{G}_{n}}(\mathrm{k} ; R)\right) \longrightarrow \mathrm{H}_{i-1}\left(\mathbf{F G}_{n}^{2}(\mathrm{k}) ; \operatorname{Res}_{\mathbf{F G}_{n}^{2}(\mathrm{k})}^{\mathbf{G}_{n}(\mathrm{k})} \operatorname{St}_{\mathbf{G}_{n}}(\mathrm{k} ; R)\right) .
$$

To prove Lemma 6.1, it is enough to prove that $\partial$ is surjective. 
We can describe $\partial$ using the recipe described in [Br2, Chapter VII.8]. Recall that $\mathbf{F G}_{n}^{3}(\mathrm{k})$ is the $\mathbf{G}_{n}(\mathrm{k})$-stabilizer of the ordered sequence of vectors $\sigma=\left[\vec{a}_{1}, \vec{a}_{2}, \vec{a}_{3}\right]$. For $1 \leq m \leq 3$, let $\sigma_{m}$ be the ordered sequence obtained by deleting $\vec{a}_{m}$ from $\sigma$ and let $\left(\mathbf{G}_{n}(k)\right)_{\sigma_{m}}$ denote the $\mathbf{G}_{n}(k)$-stabilizer of $\sigma_{m}$. We then have $\partial=\partial_{1}-\partial_{2}+\partial_{3}$, where $\partial_{m}$ is the composition

$$
\begin{aligned}
& \mathrm{H}_{i-1}\left(\mathbf{F G}_{n}^{3}(\mathrm{k}) ; \operatorname{Res}_{\mathbf{F G}_{n}^{3}(\mathrm{k})}^{\mathbf{G}_{n}(\mathrm{k})} \operatorname{St}_{\mathbf{G}_{n}}(\mathrm{k} ; R)\right) \\
& \quad \stackrel{\partial_{m}^{\prime}}{\longrightarrow} \mathrm{H}_{i-1}\left(\left(\mathbf{G}_{n}(\mathrm{k})\right)_{\sigma_{m}} ; \operatorname{Res}_{\left(\mathbf{G}_{n}(\mathrm{k})\right)_{\sigma_{m}}}^{\mathbf{G}_{n}(\mathrm{k})} \operatorname{St}_{\mathbf{G}_{n}}(\mathrm{k} ; R)\right) \\
& \quad \stackrel{\partial_{m}^{\prime \prime}}{\longrightarrow} \mathrm{H}_{i-1}\left(\mathbf{F G}_{n}^{2}(\mathrm{k}) ; \operatorname{Res}_{\mathbf{F G}_{n}(\mathrm{k})}^{\mathbf{G}_{n}(\mathrm{k})} \operatorname{St}_{\mathbf{G}_{n}}(\mathrm{k} ; R)\right)
\end{aligned}
$$

of the following two maps.

- $\partial_{m}^{\prime}$ is the map induced by the inclusion $\mathbf{F G}_{n}^{3}(\mathrm{k}) \hookrightarrow\left(\mathbf{G}_{n}(\mathrm{k})\right)_{\sigma_{m}}$.

- Define $\kappa_{m} \in \mathbf{G}_{n}(\mathrm{k})$ as follows. First, $\kappa_{3}=$ id. For $m \in\{1,2\}$, we do the following.

- If $\mathbf{G}_{n}=\mathrm{GL}_{n}$ or $\mathbf{G}_{n}=\mathrm{SL}_{n}$, then $\kappa_{m} \in \mathrm{SL}_{n}(\mathrm{k})$ is the map k $\mathrm{k}^{n} \rightarrow \mathrm{k}^{n}$ that takes $\vec{a}_{m}$ to $\vec{a}_{3}$, takes $\vec{a}_{3}$ to $-\vec{a}_{m}$, and fixes all the other basis vectors.

- If $\mathbf{G}_{n}=\mathrm{Sp}_{2 n}$ or $\mathbf{G}_{n}=\mathrm{SO}_{n, n}$ or $\mathbf{G}_{n}=\mathrm{SO}_{n, n+1}$, then $\kappa_{m} \in \mathbf{G}_{n}(\mathrm{k})$ is the map k $\mathrm{k}^{m} \rightarrow$ $\mathrm{k}^{m}$ defined as follows. Let $\vec{b}_{1}, \ldots, \vec{b}_{n}$ be the standard basis vectors for $\mathrm{k}^{m}$ that pair with the $\vec{a}_{j}$ (there is one additional standard basis vector if $\mathbf{G}_{n}=\mathrm{SO}_{n, n+1}$ ). Then $\kappa_{m}$ takes $\vec{a}_{m}$ to $\vec{a}_{3}$, takes $\vec{a}_{3}$ to $-\vec{a}_{m}$, takes $\vec{b}_{m}$ to $\vec{b}_{3}$, takes $\vec{b}_{3}$ to $-\vec{b}_{m}$, and fixes all the other basis vectors.

Then $\partial_{m}^{\prime \prime}$ is induced by the map $\left(\mathbf{G}_{n}(\mathrm{k})\right)_{\sigma_{m}} \rightarrow \mathbf{F G}_{n}^{2}(\mathrm{k})$ that takes $g \in\left(\mathbf{G}_{n}(\mathrm{k})\right)_{\sigma_{m}}$ to $\kappa_{m} g \kappa_{m}^{-1}$ and the map $\operatorname{St}_{\mathbf{G}_{n}}(\mathrm{k} ; R) \rightarrow \mathrm{St}_{\mathbf{G}_{n}}(\mathrm{k} ; R)$ that takes $x \in \mathrm{St}_{\mathbf{G}_{n}}(\mathrm{k} ; R)$ to $\kappa_{m}(x) \in$ $\mathrm{St}_{\mathbf{G}_{n}}(\mathrm{k} ; R)$. We remark that easier choices of $\kappa_{m}$ (without the signs) could be used for

$\mathbf{G}_{n} \neq \mathrm{SL}_{n}$, but we chose the ones above to make our later formulas more uniform.

This is summarized in the following lemma.

Lemma 6.2. Let the notation be as above. Then the map $\partial$ in (6.2) equals $\partial_{1}-\partial_{2}+\partial_{3}$, where $\partial_{m}$ is induced by the map $\mathbf{F G}_{n}^{3}(\mathrm{k}) \rightarrow \mathbf{F G}_{n}^{2}(\mathrm{k})$ defined via the formula

$$
g \mapsto \kappa_{m} g \kappa_{m}^{-1} \quad\left(g \in \mathbf{F G}_{n}^{3}(\mathrm{k})\right)
$$

and the map $\mathrm{St}_{\mathbf{G}_{n}}(\mathrm{k} ; R) \rightarrow \mathrm{St}_{\mathbf{G}_{n}}(\mathrm{k} ; R)$ defined via the formula

$$
x \mapsto \kappa_{m}(x) \quad\left(x \in \mathrm{St}_{\mathbf{G}_{n}}(\mathrm{k} ; R)\right) .
$$

\subsection{Bringing in the stabilization map}

The notation is as in the beginning of $\S 6$. Fix some $1 \leq m \leq 3$, and let $\partial_{m}$ and $\kappa_{m}$ be as in Lemma 6.2. Applying the isomorphism in Corollary 4.2 to the domain and codomain of $\partial_{m}$, we obtain a homomorphism

$$
\begin{aligned}
& \widehat{\partial}_{m}: \mathrm{St}_{\mathrm{GL}_{3}}(\mathrm{k} ; R) \otimes \mathrm{H}_{i-1}\left(\mathbf{G}_{n-3}(\mathrm{k}) ; \mathrm{St}_{\mathbf{G}_{n-3}}(\mathrm{k} ; R)\right) \\
& \quad \rightarrow \mathrm{St}_{\mathrm{GL}_{2}}(\mathrm{k} ; R) \otimes \mathrm{H}_{i-1}\left(\mathbf{G}_{n-2}(\mathrm{k}) ; \mathrm{St}_{\mathbf{G}_{n-2}}(\mathrm{k} ; R)\right) .
\end{aligned}
$$

Our goal in this section is to prove that $\widehat{\partial}_{m}$ is the tensor product of the stabilization map

$$
\mathrm{H}_{i-1}\left(\mathbf{G}_{n-3}(\mathrm{k}) ; \mathrm{St}_{\mathbf{G}_{n-3}}(\mathrm{k} ; R)\right) \rightarrow \mathrm{H}_{i-1}\left(\mathbf{G}_{n-2}(\mathrm{k}) ; \mathrm{St}_{\mathbf{G}_{n-2}}(\mathrm{k} ; R)\right)
$$


with the map $\zeta_{m}: \operatorname{St}_{\mathrm{GL}_{3}}(\mathrm{k} ; R) \rightarrow \mathrm{St}_{\mathrm{GL}_{2}}(\mathrm{k} ; R)$ defined as follows. Let $\left\{\vec{a}_{1}, \vec{a}_{2}, \vec{a}_{3}\right\}$ be the standard basis for $\mathrm{k}^{3}$. Let $\widehat{\kappa}_{3}=\mathrm{id} \in \mathrm{SL}_{3}(\mathrm{k})$, and for $m \in\{1,2\}$ let $\widehat{\kappa}_{m} \in \mathrm{SL}_{3}(\mathrm{k})$ be the element that takes $\vec{a}_{m}$ to $\vec{a}_{3}$, takes $\vec{a}_{3}$ to $-\vec{a}_{m}$, and fixes all the other basis vectors. Then $\zeta_{m}$ is the composition

$$
\mathrm{St}_{\mathrm{GL}_{3}}(\mathrm{k} ; R) \stackrel{\widehat{\kappa}_{m}}{\longrightarrow} \mathrm{St}_{\mathrm{GL}_{3}}(\mathrm{k} ; R) \longrightarrow \mathrm{St}_{\mathrm{GL}_{2}}(\mathrm{k} ; R) \otimes \mathrm{St}_{\mathrm{GL}_{1}}(\mathrm{k} ; R) \cong \operatorname{St}_{\mathrm{GL}_{2}}(\mathrm{k} ; R),
$$

where the second arrow is the Reeder projection map (see §4) and the final isomorphism comes from the fact that $\mathrm{St}_{\mathrm{GL}_{1}}(\mathrm{k} ; R)=R$.

The main result of this section is then as follows.

Lemma 6.3. Let the notation be as above. Then $\widehat{\partial}_{m}$ is the tensor product of $\zeta_{m}$ with the stabilization map (6.3).

Proof. By construction, $\widehat{\partial}_{m}$ equals the composition

$$
\begin{aligned}
& \mathrm{St}_{\mathrm{GL}_{3}}(\mathrm{k} ; R) \otimes \mathrm{H}_{i-1}\left(\mathbf{G}_{n-3}(\mathrm{k}) ; \mathrm{St}_{\mathbf{G}_{n-3}}(\mathrm{k} ; R)\right) \\
& \stackrel{\cong}{\longrightarrow} \mathrm{H}_{i-1}\left(1 \times \mathbf{G}_{n-3}(\mathrm{k}) ; \operatorname{St}_{\mathrm{GL}_{3}}(\mathrm{k} ; R) \otimes \operatorname{St}_{\mathbf{G}_{n-3}}(\mathrm{k} ; R)\right) \\
& \stackrel{\cong}{\longrightarrow} \mathrm{H}_{i-1}\left(\mathbf{F G}_{n}^{3}(\mathrm{k}) ; \mathrm{St}_{\mathbf{G}_{n}}(\mathrm{k} ; R)\right) \\
& \stackrel{\partial_{m}}{\longrightarrow} \mathrm{H}_{i-1}\left(\mathbf{F G}_{n}^{2}(\mathrm{k}) ; \mathrm{St}_{\mathbf{G}_{n}}(\mathrm{k} ; R)\right) \\
& \stackrel{\cong}{\longrightarrow} \mathrm{H}_{i-1}\left(1 \times \mathbf{G}_{n-2}(\mathrm{k}) ; \operatorname{St}_{\mathrm{GL}_{2}}(\mathrm{k} ; R) \otimes \operatorname{St}_{\mathbf{G}_{n-2}}(\mathrm{k} ; R)\right) \\
& \stackrel{\cong}{\longrightarrow} \operatorname{St}_{\mathrm{GL}_{2}}(\mathrm{k} ; R) \otimes \mathrm{H}_{i-1}\left(\mathbf{G}_{n-2}(\mathrm{k}) ; \mathrm{St}_{\mathbf{G}_{n-2}}(\mathrm{k} ; R)\right)
\end{aligned}
$$

where the various maps are as follows:

- The first and last arrows use the fact that $\mathrm{St}_{\mathrm{GL}_{3}}(\mathrm{k} ; R)$ and $\mathrm{St}_{\mathrm{GL}_{2}}(\mathrm{k} ; R)$ are free $R$ modules (cf. the proof of Corollary 4.2).

- The second arrow is the map described in Lemma 4.1, that is, the map induced by the inclusion $1 \times \mathbf{G}_{n-3}(\mathrm{k}) \hookrightarrow \mathbf{F G}_{n}^{3}(\mathrm{k})$ and the Reeder product map $\operatorname{St}_{\mathrm{GL}_{3}}(\mathrm{k} ; R) \otimes$ $\mathrm{St}_{\mathbf{G}_{n-3}}(\mathrm{k} ; R) \rightarrow \mathrm{St}_{\mathbf{G}_{n}}(\mathrm{k} ; R)$.

- The third arrow is the map $\partial_{m}$ described in Lemma 6.2, that is, the map induced by the map $\mathbf{F G}_{n}^{3}(\mathrm{k}) \rightarrow \mathbf{F G}_{n}^{2}(\mathrm{k})$ given by conjugation by $\kappa_{m}$ and the map $\operatorname{St}_{\mathbf{G}_{n}}(\mathrm{k} ; R) \rightarrow$ $\mathrm{St}_{\mathbf{G}_{n}}(\mathrm{k} ; R)$ induced by $\kappa_{m}$.

- The fourth arrow is the map described in Lemma 4.3, that is, the map induced by the projection $\mathbf{F G}_{n}^{2}(\mathrm{k}) \rightarrow 1 \times \mathbf{G}_{n-2}(\mathrm{k})$ together with the Reeder projection map $\mathrm{St}_{\mathbf{G}_{n}}(\mathrm{k} ; R) \rightarrow \mathrm{St}_{\mathrm{GL}_{2}}(\mathrm{k} ; R) \otimes \mathrm{St}_{\mathbf{G}_{n-2}}(\mathrm{k} ; R)$.

We must show that this composition equals the indicated tensor product of maps. This will take some work.

Define $\Psi$ to be the composition

$$
\begin{aligned}
\mathrm{St}_{\mathrm{GL}_{3}}(\mathrm{k} ; R) \otimes \mathrm{St}_{\mathbf{G}_{n-3}}(\mathrm{k} ; R) & \longrightarrow \mathrm{St}_{\mathbf{G}_{n}}(\mathrm{k} ; R) \\
& \stackrel{\kappa_{m}}{\longrightarrow} \mathrm{St}_{\mathbf{G}_{n}}(\mathrm{k} ; R) \\
& \longrightarrow \mathrm{St}_{\mathrm{GL}_{2}}(\mathrm{k} ; R) \otimes \mathrm{St}_{\mathbf{G}_{n-2}}(\mathrm{k} ; R),
\end{aligned}
$$


where the first map is the Reeder product map and the last map is the Reeder projection map. Also, define $\Phi$ to be the composition

$$
\begin{aligned}
\mathrm{St}_{\mathrm{GL}_{3}}(\mathrm{k} ; R) \otimes \mathrm{St}_{\mathbf{G}_{n-3}}(\mathrm{k} ; R) & \longrightarrow \mathrm{St}_{\mathrm{GL}_{2}}(\mathrm{k} ; R) \otimes \mathrm{St}_{\mathrm{GL}_{1}}(\mathrm{k} ; R) \otimes \mathrm{St}_{\mathbf{G}_{n-3}}(\mathrm{k} ; R) \\
& \longrightarrow \mathrm{St}_{\mathrm{GL}_{2}}(\mathrm{k} ; R) \otimes \mathrm{St}_{\mathbf{G}_{n-2}}(\mathrm{k} ; R),
\end{aligned}
$$

where the maps are as follows:

- The first map is the tensor product of the Reeder projection map $\operatorname{St}_{\mathrm{GL}_{3}}(\mathrm{k} ; R) \rightarrow$ $\mathrm{St}_{\mathrm{GL}_{2}}(\mathrm{k} ; R) \otimes \mathrm{St}_{\mathrm{GL}_{1}}(\mathrm{k} ; R)$ and the identity $\operatorname{map} \operatorname{St}_{\mathbf{G}_{n-3}}(\mathrm{k} ; R) \rightarrow \mathrm{St}_{\mathbf{G}_{n-3}}(\mathrm{k} ; R)$.

- The second map is the tensor product of the identity map $\operatorname{St}_{\mathrm{GL}_{2}}(\mathrm{k} ; R) \rightarrow \mathrm{St}_{\mathrm{GL}_{2}}(\mathrm{k} ; R)$ and the Reeder product map $\operatorname{St}_{\mathrm{GL}_{1}}(\mathrm{k} ; R) \otimes \mathrm{St}_{\mathbf{G}_{n-3}}(\mathrm{k} ; R) \rightarrow \mathrm{St}_{\mathbf{G}_{n-2}}(\mathrm{k} ; R)$.

By the above, it is enough to prove that $\Psi=\Phi \circ\left(\widehat{\kappa}_{m} \otimes \mathrm{id}\right)$.

Define $\Psi^{\prime}$ to be the composition

$$
\begin{aligned}
\operatorname{St}_{\mathrm{GL}_{3}}(\mathrm{k} ; R) \otimes \mathrm{St}_{\mathbf{G}_{n-3}}(\mathrm{k} ; R) & \longrightarrow \mathrm{St}_{\mathbf{G}_{n}}(\mathrm{k} ; R) \\
& \longrightarrow \mathrm{St}_{\mathrm{GL}_{2}}(\mathrm{k} ; R) \otimes \mathrm{St}_{\mathbf{G}_{n-2}}(\mathrm{k} ; R),
\end{aligned}
$$

where the first map is the Reeder product map and the second map is the Reeder projection map. From its definition, we see that $\Psi=\Psi^{\prime} \circ\left(\widehat{\kappa}_{m} \otimes \mathrm{id}\right)$. We thus see that it is enough to prove that $\Psi^{\prime}=\Phi$.

Define $U=\mathbf{U G L}_{3}^{2}(\mathrm{k})$ to be the unipotent radical of the parabolic subgroup $\mathbf{P G L}_{3}^{2}(\mathrm{k})$ of $\mathrm{GL}_{3}(\mathrm{k})$ (despite the bad notation, this is not the projective general linear group). Using Theorem 2.1 as in Remark 2.5, we see that

$$
\mathrm{St}_{\mathrm{GL}_{3}}(\mathrm{k} ; R)=\bigoplus_{u \in U} u \cdot\left(\mathrm{St}_{\mathrm{GL}_{2}}(\mathrm{k} ; R) \otimes \mathrm{St}_{\mathrm{GL}_{1}}(\mathrm{k} ; R)\right) .
$$

Consider $u \in U$ and $x \in \operatorname{St}_{\mathrm{GL}_{2}}(\mathrm{k} ; R)$ and $y \in \operatorname{St}_{\mathrm{GL}_{1}}(\mathrm{k} ; R)$ and $z \in \operatorname{St}_{\mathbf{G}_{n-3}}(\mathrm{k} ; R)$. Examining the definition of $\Psi^{\prime}$, we see that

$$
\Psi^{\prime}((u \cdot(x \otimes y)) \otimes z)=x \otimes(y \otimes z),
$$

where $y \otimes z \in \operatorname{St}_{\mathrm{GL}_{1}}(\mathrm{k} ; R) \otimes \operatorname{St}_{\mathbf{G}_{n-3}}(\mathrm{k} ; R)$ is identified with an element of $\operatorname{St}_{\mathbf{G}_{n-2}}(\mathrm{k} ; R)$ using the Reeder product map. But this equals $\Phi((u \cdot(x \otimes y)) \otimes z)$, as desired.

\subsection{Summary of where we are}

The notation is as in the beginning of $\S 6$. Recall that Lemma 6.1 asserts that the differential $E_{2, i-1}^{1} \rightarrow E_{1, i-1}^{1}$ is surjective. Let $\partial$ be as in $\S 6.1$. Also, let $\zeta_{m}$ and $\widehat{\kappa}_{m}$ be as in $\$ 6.2$. Define

$$
\zeta: \mathrm{St}_{\mathrm{GL}_{3}}(\mathrm{k} ; R) \rightarrow \mathrm{St}_{\mathrm{GL}_{2}}(\mathrm{k} ; R)
$$

via the formula $\zeta=\zeta_{1}-\zeta_{2}+\zeta_{3}$. Combining Lemmas 6.2 and 6.3 , we see that to prove Lemma 6.1, it is enough to show that the map

$$
\mathrm{St}_{\mathrm{GL}_{3}}(\mathrm{k} ; R) \otimes \mathrm{H}_{i-1}\left(\mathbf{G}_{n-3}(\mathrm{k}) ; \mathrm{St}_{\mathbf{G}_{n-3}}(\mathrm{k} ; R)\right) \rightarrow \mathrm{St}_{\mathrm{GL}_{2}}(\mathrm{k} ; R) \otimes \mathrm{H}_{i-1}\left(\mathbf{G}_{n-2}(\mathrm{k}) ; \operatorname{St}_{\mathbf{G}_{n-2}}(\mathrm{k} ; R)\right)
$$

obtained as the tensor product of $\zeta$ and the stabilization map

$$
\mathrm{H}_{i-1}\left(\mathbf{G}_{n-3}(\mathrm{k}) ; \operatorname{St}_{\mathbf{G}_{n-3}}(\mathrm{k} ; R)\right) \rightarrow \mathrm{H}_{i-1}\left(\mathbf{G}_{n-2}(\mathrm{k}) ; \operatorname{St}_{\mathbf{G}_{n-2}}(\mathrm{k} ; R)\right)
$$

is surjective. One of the assumptions in Lemma 6.1 is that this stabilization map is surjective. To prove that lemma, it is thus enough to prove the following.

Lemma 6.4. Let the notation be as above. Then $\zeta$ is surjective. 


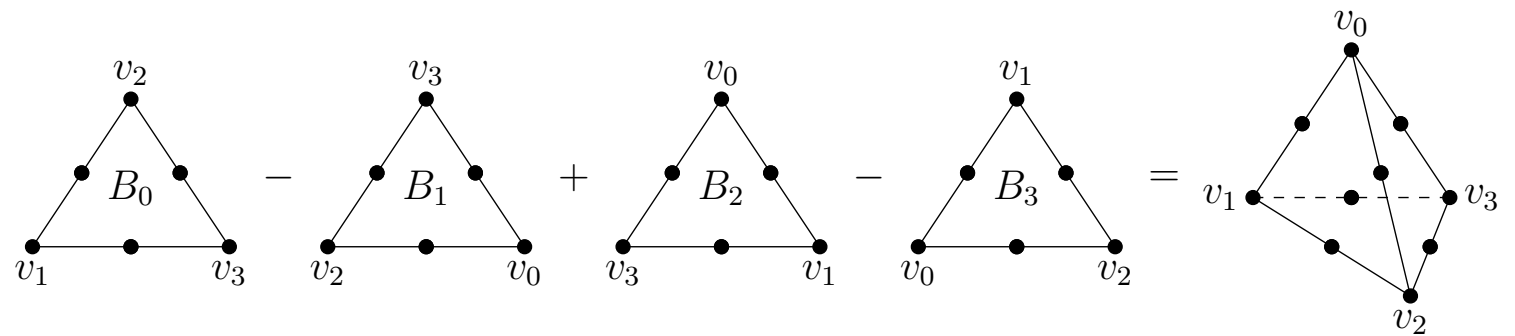

Figure 1: As we illustrate here in the case $n=3$, the apartment classes corresponding to the $B_{i}$ can be placed on the boundary of an n-dimensional simplex such that their simplices cancel in pairs. In the picture, the vertices labeled with the vectors $\vec{v}_{i}$ are taken to the lines spanned by the $\vec{v}_{i}$ while the unlabeled vertices are taken to the 2-dimensional subspaces spanned by the vectors on their two neighbors.

\subsection{Apartments}

Before we prove Lemma 6.4, we need to discuss some background material on the Steinberg representation. Unlike the previous sections, in this section $n \geq 1$ is arbitrary. Recall that $\operatorname{St}_{\mathrm{GL}_{n}}(\mathrm{k} ; R)=\widetilde{\mathrm{H}}_{n-2}\left(\mathcal{T}_{\mathrm{GL}_{n}}(\mathrm{k}) ; R\right)$, where $\mathcal{T}_{\mathrm{GL}_{n}}(\mathrm{k})$ is the Tits building associated to $\mathrm{GL}_{n}(\mathrm{k})$. This building can be described as the simplicial complex whose $r$-simplices are flags

$$
0 \subsetneq V_{0} \subsetneq \cdots \subsetneq V_{r} \subsetneq \mathrm{k}^{n}
$$

of nonzero proper subspaces of $\mathrm{k}^{n}$.

The Solomon-Tits theorem [Br1, Theorem IV.5.2] says that the $R$-module $\mathrm{St}_{\mathrm{GL}_{n}}(\mathrm{k} ; R)$ is spanned by apartment classes, which are defined as follows. Consider an $n \times n$ matrix $B$ with entries in $\mathrm{k}^{n}$ none of whose columns are identically 0 . Let $\left(\vec{v}_{1}, \ldots, \vec{v}_{n}\right)$ be the columns of $B$. Let $S_{n}$ be the simplicial complex whose $r$-simplices are chains

$$
0 \subsetneq I_{0} \subsetneq \cdots \subsetneq I_{r} \subsetneq\{1, \ldots, n\} .
$$

The complex $S_{n}$ is isomorphic to the barycentric subdivision of the boundary of an $(n-1)$ simplex; in particular, $S_{n}$ is homeomorphic to an $(n-2)$-sphere. There is a simplicial map $f: S_{n} \rightarrow \mathcal{T}_{\mathrm{GL}_{n}}(\mathrm{k})$ defined via the formula

$$
f(I)=\left\langle\vec{v}_{i} \mid i \in I\right\rangle \quad(\emptyset \subsetneq I \subsetneq\{1, \ldots, n\}) .
$$

The apartment class corresponding to $B$, denoted $\|B\|$, is the image of the fundamental class $\left[S_{n}\right] \in \widetilde{\mathrm{H}}_{n-2}\left(S_{n} ; R\right)=R$ under the map $f_{*}: \widetilde{\mathrm{H}}_{n-2}\left(S_{n} ; R\right) \rightarrow \widetilde{\mathrm{H}}_{n-2}\left(\mathcal{T}_{\mathrm{GL}_{n}}(\mathrm{k}) ; R\right)=\operatorname{St}_{\mathrm{GL}_{n}}(\mathrm{k} ; R)$.

Remark 6.5. We have $\|B\|=0$ if the $\vec{v}_{i}$ do not form a basis for $\mathrm{k}^{n}$, i.e. if $B$ is not invertible.

Permuting the columns of $B$ changes $\|B\|$ by the sign of the permutation, and multiplying a column of $B$ by a nonzero scalar does not change $\|B\|$. The apartment classes also satisfy the following more interesting relation. 
Lemma 6.6. Let $\mathrm{k}$ be a field, let $R$ be a commutative ring, and let $n \geq 2$. Let $B$ be an $n \times(n+1)$-matrix with entries in $\mathrm{k}$. Assume that none of the columns of $B$ are identically 0 . Ordering the columns of $B$ from 0 to $n$, for $0 \leq m \leq n$ let $B_{m}$ be the result of deleting the $m^{\text {th }}$ column from $B$. Then $\left\|B_{0}\right\|-\left\|B_{1}\right\|+\left\|B_{2}\right\|-\cdots+(-1)^{n}\left\|B_{n}\right\|=0$.

Proof. The simplices forming the apartment classes $\left\|B_{i}\right\|$ cancel in pairs; see Figure 1.

The Solomon-Tits theorem [Br1, Theorem IV .5.2] gives the following basis for $\operatorname{St}_{\mathrm{GL}_{n}}(\mathrm{k} ; R)$.

Theorem 6.7 (Solomon-Tits). Let $\mathrm{k}$ be a field, let $R$ be a commutative ring, and let $n \geq 1$. Then $\mathrm{St}_{\mathrm{GL}_{n}}(\mathrm{k} ; R)$ is a free $R$-module on the basis consisting of all $\|B\|$ such that $B$ is an upper unitriangular matrix in $\mathrm{GL}_{n}(\mathrm{k})$.

\subsection{The proof of Lemma 6.4}

We finally prove Lemma 6.4, which as discussed in $\S 6.3$ suffices to prove Lemma 6.1. First, we recall its statement. For $1 \leq m \leq 3$, let $\zeta_{m}$ and $\widehat{\kappa}_{m}$ be as in $\S 6.2$. Define

$$
\zeta: \mathrm{St}_{\mathrm{GL}_{3}}(\mathrm{k} ; R) \rightarrow \mathrm{St}_{\mathrm{GL}_{2}}(\mathrm{k} ; R)
$$

via the formula $\zeta=\zeta_{1}-\zeta_{2}+\zeta_{3}$. Our goal is to prove that $\zeta$ is surjective.

Before we do that, we introduce some formulas. Let $\pi: \mathrm{St}_{\mathrm{GL}_{3}}(\mathrm{k} ; R) \rightarrow \mathrm{St}_{\mathrm{GL}_{2}}(\mathrm{k} ; R)$ be the composition

$$
\mathrm{St}_{\mathrm{GL}_{3}}(\mathrm{k} ; R) \longrightarrow \mathrm{St}_{\mathrm{GL}_{2}}(\mathrm{k} ; R) \otimes \mathrm{St}_{\mathrm{GL}_{1}}(\mathrm{k} ; R) \stackrel{\cong}{\longrightarrow} \operatorname{St}_{\mathrm{GL}_{2}}(\mathrm{k} ; R),
$$

where the first arrow is the Reeder projection map and the second arrow comes from the fact that $\mathrm{St}_{\mathrm{GL}_{1}}(\mathrm{k} ; R)=R$. From its definition, we see that

$$
\pi\left(\left\|\begin{array}{ccc}
1 & x & y \\
0 & 1 & z \\
0 & 0 & 1
\end{array}\right\|\right)=\left\|\begin{array}{ll}
1 & x \\
0 & 1
\end{array}\right\|
$$

for all $x, y, z \in \mathrm{k}$. What is more, for all $3 \times 3$ matrices $B$ none of whose columns are identically 0 we have

$$
\zeta(\|B\|)=\pi\left(\widehat{\kappa}_{1}(B)\right)-\pi\left(\widehat{\kappa}_{2}(B)\right)+\pi\left(\widehat{\kappa}_{3}(B)\right) .
$$

Here the $\widehat{\kappa}_{m}$ act on $B$ via matrix multiplication.

We now turn to proving that $\zeta$ is surjective. Consider $a \in \mathrm{k}$, and set

$$
A_{a}=\left\|\begin{array}{cc}
1 & a \\
0 & 1
\end{array}\right\| .
$$


By Theorem 6.7, it is enough to prove that $A_{a} \in \operatorname{Im}(\zeta)$. We have

$$
\begin{aligned}
\zeta\left(\left\|\begin{array}{lll}
1 & a & 0 \\
0 & 1 & 0 \\
0 & 0 & 1
\end{array}\right\|\right) & =\pi\left(\left\|\begin{array}{ccc}
0 & 0 & -1 \\
0 & 1 & 0 \\
1 & a & 0
\end{array}\right\|\right)-\pi\left(\left\|\begin{array}{ccc}
1 & a & 0 \\
0 & 0 & -1 \\
0 & 1 & 0
\end{array}\right\|\right)+\pi\left(\left\|\begin{array}{ccc}
1 & a & 0 \\
0 & 1 & 0 \\
0 & 0 & 1
\end{array}\right\|\right) \\
& =\pi\left(-\left\|\begin{array}{ccc}
-1 & 0 & 0 \\
0 & 1 & 0 \\
0 & a & 1
\end{array}\right\|\right)-\pi\left(-\left\|\begin{array}{ccc}
1 & 0 & a \\
0 & -1 & 0 \\
0 & 0 & 1
\end{array}\right\|\right)+\pi\left(\left\|\begin{array}{ccc}
1 & a & 0 \\
0 & 1 & 0 \\
0 & 0 & 1
\end{array}\right\|\right) \\
& =\pi\left(-\left\|\begin{array}{lll}
1 & 0 & 0 \\
0 & 1 & 0 \\
0 & a & 1
\end{array}\right\|\right)-\pi\left(-\left\|\begin{array}{ccc}
1 & 0 & a \\
0 & 1 & 0 \\
0 & 0 & 1
\end{array}\right\|\right)+\pi\left(\left\|\begin{array}{ccc}
1 & a & 0 \\
0 & 1 & 0 \\
0 & 0 & 1
\end{array}\right\|\right) \\
& =-\pi\left(\left\|\begin{array}{lll}
1 & 0 & 0 \\
0 & 1 & 0 \\
0 & a & 1
\end{array}\right\|\right)+\left\|\begin{array}{ll}
1 & 0 \\
0 & 1
\end{array}\right\|+\left\|\begin{array}{ll}
1 & a \\
0 & 1
\end{array}\right\|,
\end{aligned}
$$

where the second equality uses the fact that permuting the columns of a matrix changes the associated apartment by the sign of the permutation and the third equality uses the fact that multiplying a column by a nonzero scalar does not change the associated apartment.

If $a=0$, then the right hand side of (6.4) simplifies to

$$
-\left\|\begin{array}{ll}
1 & 0 \\
0 & 1
\end{array}\right\|+\left\|\begin{array}{ll}
1 & 0 \\
0 & 1
\end{array}\right\|+\left\|\begin{array}{ll}
1 & 0 \\
0 & 1
\end{array}\right\|=\left\|\begin{array}{ll}
1 & 0 \\
0 & 1
\end{array}\right\|=A_{0},
$$

so $A_{0} \in \operatorname{Im}(\zeta)$. Assume now that $a \neq 0$. Plugging the matrix

$$
\left(\begin{array}{llll}
1 & 0 & 0 & 0 \\
0 & 1 & 1 & 0 \\
0 & 0 & a & 1
\end{array}\right)
$$

into Lemma 6.6, we get the relation

$$
\begin{aligned}
0 & =\left\|\begin{array}{lll}
0 & 0 & 0 \\
1 & 1 & 0 \\
0 & a & 1
\end{array}\right\|-\|\| \begin{array}{ccc}
1 & 0 & 0 \\
0 & 1 & 0 \\
0 & a & 1
\end{array}\|+\| \begin{array}{ccc}
1 & 0 & 0 \\
0 & 1 & 0 \\
0 & 0 & 1
\end{array}\|-\| \begin{array}{ccc}
1 & 0 & 0 \\
0 & 1 & 1 \\
0 & 0 & a
\end{array} \| \\
& =0-\left\|\begin{array}{lll}
1 & 0 & 0 \\
0 & 1 & 0 \\
0 & a & 1
\end{array}\right\|+\left\|\begin{array}{ccc}
1 & 0 & 0 \\
0 & 1 & 0 \\
0 & 0 & 1
\end{array}\right\|-\left\|\begin{array}{ccc}
1 & 0 & 0 \\
0 & 1 & a^{-1} \\
0 & 0 & 1
\end{array}\right\|,
\end{aligned}
$$

where the equality uses the fact that the columns of the first matrix are not linearly independent and the fact that multiplying a column of a matrix by a nonzero scalar does not change the associated apartment. Plugging this relation into (6.4), we see that the right hand side of (6.4) equals

$$
\begin{aligned}
& -\left(\pi\left(\left\|\begin{array}{lll}
1 & 0 & 0 \\
0 & 1 & 0 \\
0 & 0 & 1
\end{array}\right\|\right)-\pi\left(\left\|\begin{array}{ccc}
1 & 0 & 0 \\
0 & 1 & a^{-1} \\
0 & 0 & 1
\end{array}\right\|\right)+\left\|\begin{array}{ll}
1 & 0 \\
0 & 1
\end{array}\right\|+\left\|\begin{array}{cc}
1 & a \\
0 & 1
\end{array}\right\|\right. \\
= & -\left\|\begin{array}{ll}
1 & 0 \\
0 & 1
\end{array}\right\|+\left\|\begin{array}{ll}
1 & 0 \\
0 & 1
\end{array}\right\|+\left\|\begin{array}{ll}
1 & 0 \\
0 & 1
\end{array}\right\|+\left\|\begin{array}{cc}
1 & a \\
0 & 1
\end{array}\right\| \\
= & A_{0}+A_{a} .
\end{aligned}
$$


Since we have already seen that $A_{0} \in \operatorname{Im}(\zeta)$, we deduce that $A_{a} \in \operatorname{Im}(\zeta)$, as desired.

\section{References}

[BoSe] A. Borel and J.-P. Serre, Corners and arithmetic groups, Comment. Math. Helv. 48 (1973), 436-491.

[Br1] K. S. Brown, Buildings, Springer, New York, 1989.

[Br2] K. S. Brown, Cohomology of groups, corrected reprint of the 1982 original, Graduate Texts in Mathematics, 87, Springer, New York, 1994.

[CFaP $] \quad$ T. Church, B. Farb and A. Putman, A stability conjecture for the unstable cohomology of $\mathrm{SL}_{n} \mathbb{Z}$, mapping class groups, and $\operatorname{Aut}\left(F_{n}\right)$, in Algebraic topology: applications and new directions, 55-70, Contemp. Math., 620, Amer. Math. Soc., Providence, RI, arXiv: $1208.3216 \mathrm{v} 3$.

[CP] T. Church and A. Putman, The codimension-one cohomology of $\mathrm{SL}_{n} \mathbb{Z}$, Geom. Topol., to appear, arXiv:1507.06306v3.

[Dw] W. G. Dwyer, Twisted homological stability for general linear groups, Ann. of Math. (2) 111 (1980), no. 2, 239-251.

[Fr] N. Friedrich, Homological Stability of automorphism groups of quadratic modules and manifolds, preprint 2016, arXiv:1612.04584v2.

[H] J. E. Humphreys, The Steinberg representation, Bull. Amer. Math. Soc. (N.S.) 16 (1987), no. $2,247-263$.

[LSz] R. Lee and R. H. Szczarba, On the homology and cohomology of congruence subgroups, Invent. Math. 33 (1976), no. 1, 15-53.

[Maa] H. Maazen, Homology stability for the general linear group, thesis, University of Utrecht, 1979.

[Mac] I. G. Macdonald, Symmetric Functions and Hall Polynomials, second edition, Oxford Mathematical Monographs, Oxford, 1995.

[MiVdK] B. Mirzaii, W. van der Kallen, Homology stability for unitary groups, Doc. Math. 7 (2002), 143-166.

[MalZ] G. Malle and A. Zalesski, Prime power degree representations of quasi-simple groups, Archiv. Math. 77 (2001), 461-468.

[Q] D. Quillen, Finite generation of the groups $K_{i}$ of rings of algebraic integers, in Algebraic K-theory, I: Higher K-theories (Proc. Conf., Battelle Memorial Inst., Seattle, Wash., 1972), 179-198. Lecture Notes in Math., 341, Springer, Berlin.

[RaWiWa] O. Randal-Williams and N. Wahl, Homological stability for automorphism groups, preprint 2015, arXiv:1409.3541v3.

[Re] M. Reeder, The Steinberg module and the cohomology of arithmetic groups, J. Algebra 141 (1991), no. 2, 287-315.

[VdK] W. van der Kallen, Homology stability for linear groups, Invent. Math. 60 (1980), no. 3, 269-295.

Avner Ash

Department of Mathematics

Boston College

Chestnut Hill, MA 02467-3806

ashav@bc.edu
Andrew Putman

Department of Mathematics University of Notre Dame

279 Hurley Hall

Notre Dame, IN 46556

andyp@nd.edu
Steven V Sam

Department of Mathematics

University of Wisconsin

480 Lincoln Dr.

Madison, WI 53706-1325

svs@math.wisc .edu 